\title{
TRIPLE CORRELATION OF THE RIEMANN ZEROS
}

\author{
J.B. CONREY AND N.C. SNAITH
}

\begin{abstract}
We use the conjecture of Conrey, Farmer and Zirnbauer for averages of ratios of the Riemann zeta function [1] to calculate all the lower order terms of the triple correlation function of the Riemann zeros. A previous approach was suggested by Bogomolny and Keating [6] taking inspiration from semi-classical methods. At that point they did not write out the answer explicitly, so we do that here, illustrating that by our method all the lower order terms down to the constant can be calculated rigourously if one assumes the ratios conjecture of Conrey, Farmer and Zirnbauer. Bogomolny and Keating [4] returned to their previous results simultaneously with this current work, and have written out the full expression. The result presented in this paper agrees precisely with their formula, as well as with our numerical computations, which we include here.

We also include an alternate proof of the triple correlation of eigenvalues from random $U(N)$ matrices which follows a nearly identical method to that for the Riemann zeros, but is based on the theorem for averages of ratios of characteristic polynomials [12, 13.
\end{abstract}

\section{Contents}

1. Introduction

2. The Riemann zeros 4

2.1. Results 4

2.2. Moments of the logarithmic derivative of the Riemann zeta function 8

2.3. Triple correlation as a contour integral 13

2.4. Contributions from lower-order correlations $\quad 19$

2.5. Retrieving the asymptotic result 25

3. Random Matrix Theory 28

3.1. Triple correlation by Gaudin's Lemma 30

3.2. Triple correlation via the ratios theorem 30

References

Research of the first author supported by the American Institute of Mathematics. The second author was supported by an EPSRC Advanced Research Fellowship. Both authors have been supported by a Focused Research Group grant (0244660) from the National Science Foundation. 


\section{INTRODUCTION}

In 1973 Montgomery [22] proved the following result, assuming the Riemann Hypothesis, on the two-point correlation of the zeros of the Riemann zeta function:

$$
\begin{aligned}
\sum_{\gamma_{1}, \gamma_{2} \in[0, T]} & w\left(\gamma_{1}-\gamma_{2}\right) f\left(\frac{\log T}{2 \pi}\left(\gamma_{1}-\gamma_{2}\right)\right) \\
& =\frac{T \log T}{2 \pi}\left(f(0)+\int_{-\infty}^{\infty} f(u)\left[1-\left(\frac{\sin (\pi u)}{\pi u}\right)^{2}\right] d u+o(1)\right)
\end{aligned}
$$

for suitably decaying functions $f$ with Fourier transform supported in $[-1,1]$ and weight $w(x)=$ $\frac{4}{4+u^{2}}$. He conjectured that (1.1) would in fact hold for any test function $f$.

In 1994 Hejal [16] proved a similar result for the triple correlation of Riemann zeros:

$$
\begin{aligned}
& \sum_{\substack{\gamma_{1}, \gamma_{2}, \gamma_{3} \in[T, 2 T] \\
\text { distinct }}} w\left(\gamma_{1}, \gamma_{2}, \gamma_{3}\right) f\left(\frac{\log T}{2 \pi}\left(\gamma_{1}-\gamma_{2}\right), \frac{\log T}{2 \pi}\left(\gamma_{1}-\gamma_{3}\right)\right) \\
& =\frac{T \log T}{2 \pi}\left(\int_{-\infty}^{\infty} \int_{-\infty}^{\infty} f(u, v)\left|\begin{array}{ccc}
1 & S(u) & S(v) \\
S(u) & 1 & S(u-v) \\
S(v) & S(u-v) & 1
\end{array}\right| d u d v\right. \\
& +o(1))
\end{aligned}
$$

with weight $w\left(x_{1}, x_{2}, x_{3}\right)=\prod_{j<k} \exp \left[-\frac{1}{6}\left(x_{j}-x_{k}\right)^{2}\right]$ and the Fourier transform of the continuous, suitably decaying test function $f$ is supported on the hexagon with successive vertices $(1,0),(0,1)$, $(-1,1),(-1,0),(0,-1)$ and $(1,-1)$. Here $S(x)=\frac{\sin (\pi x)}{\pi x}$.

This was extended to the $n$-point correlation function and to more general $L$-functions by Rudnick and Sarnak 23] in 1996.

These results encompass the rigorous work on the subject, but are limited by two things. Firstly, the support of the Fourier transform of the test function is always confined to a restricted range. Secondly, only the asymptotic for large $T$ is found. This second point is understandable, as the goal was to show that this limiting form was the same as that for the $n$-point correlation function of eigenvalues from large-dimensional matrices from the GUE ensemble of random matrix theory (see [8] or [20] for review articles on the connection between random matrix theory and number theory). This aim was duly achieved, but there is clearly interest in the lower-order terms, as Bogomolny and Keating's early results [6] showed that in the two-point correlation function of the Riemann zeros one sees sensitivity to the positions of the low Riemann zeros themselves - something that clearly does not happen in random matrix theory at any order. After it was predicted by Bogomolny and Keating, a numerical illustration of this for the two-point correlation function was first shown in 2, where Berry and Keating also fully explain a similar phenomenon in the number variance statistic first observed by Berry in 1988 [1. A numerical plot of the two-point correlation function calculated using the first 100000 zeros of the Riemann zeta function is shown in Figure 1, The $\mathrm{x}$-axis is the correlation distance between pairs of zeros in unscaled units, showing the distinctive dip at each Riemann zero. The plot is a histogram of the number of pairs of zeros with a given 


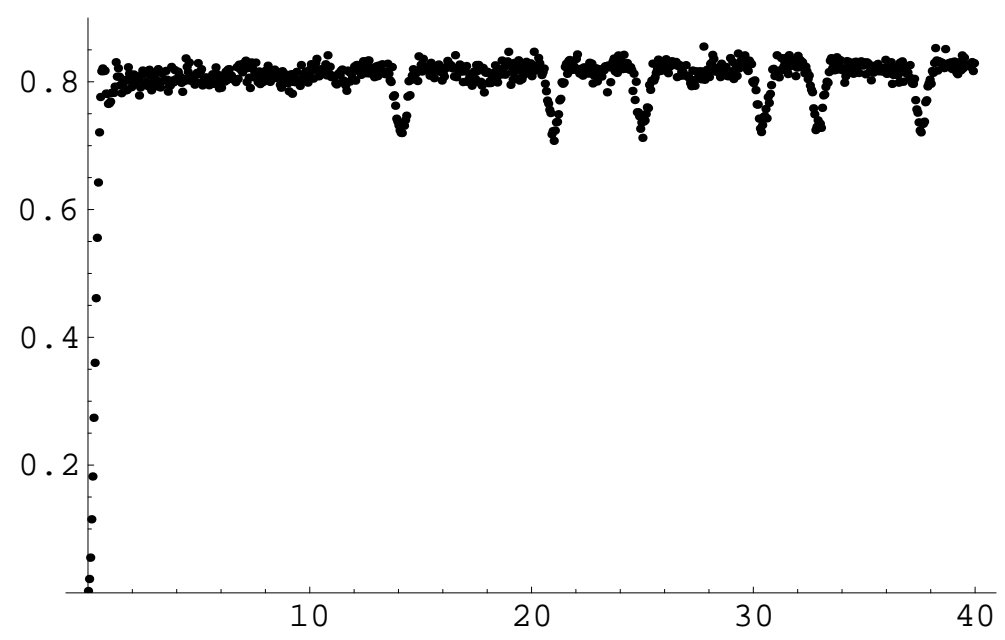

Figure 1. The two-point correlation function of the Riemann zeta function calculated from the first 100000 zeros. The correlation distance is plotted along the $\mathrm{x}$-axis. Note the significant dip at the first few Riemann zeros: 14.13, 21.02, 25.01, $\ldots$

separation distance, and the $\mathrm{y}$-axis is divided by $T\left(\frac{\log \frac{T}{2 \pi}}{2 \pi}\right)^{2}$, where $T \sim 75000$ is the height of the 100000 th zero.

Meanwhile, alongside this rigorous work in the number theory community, physicists using semiclassical techniques applied in the field of quantum chaos, treated the Riemann zeta function as a model system (the prime numbers playing the role of periodic orbits) and so studied the correlation of Riemann zeros in analogous ways to those in which they would study correlation of energy levels in their more standard physical systems. The first step in this direction was the derivation by Keating 17] of the limiting form of the two-point correlation function of the Riemann zeros. This is a heuristic calculation and it relies on a conjecture by Hardy and Littlewood [15] for the behaviour of correlations between prime numbers, but it has the advantage that there are no restrictions on the support of a test function. Using analogous methods this result was then extended in two papers by Bogomolny and Keating [5, 7] to obtain the limiting form of the $n$-point correlation function.

The first result on lower-order terms of the correlations of the Riemann zeros was also by Bogomolny and Keating [6], but using a different heuristic inspired more directly from semiclassical methods. Here they truncate the Euler product for the Riemann zeta function at primes less than $\log \frac{T}{2 \pi}$ (in semiclassical language this means taking periodic orbits up to the Heisenberg time) and define a new set of zeros from the resulting approximation to the staircase function of the Riemann zeros (the function that increases by one at the position of each zero). It is the two-point correlation of this new set of zeros that miraculously gives all the significant lower-order terms of the analogous statistic for the Riemann zeros. Using the same method, an expression is also given [6] in semiclassical language for all lower-order terms of the three-point correlation function, and it is this which could be turned into a detailed formula including all terms calculated in the present paper, but the authors did not publish it explicitly at that time. 
Bogomolny and Keating also did further work, largely unpublished, obtaining all the lower-order terms for the 2-, 3- and 4-point correlations both by extending their Hardy-Littlewood method (see 18. for some details of the two-point correlation function) and by the method mentioned in the previous paragraph, as well as two other heuristic methods (see [3]).

Recently [4] they have written out the lower-order terms for the three point correlation function of the Riemann zeros in full detail and these agree with the results presented in this paper.

\section{The Riemann zeros}

2.1. Results. Using the ratios conjecture of Conrey, Farmer and Zirnbauer [12, 11] we obtain the following

Theorem 2.1. Assuming the ratios conjecture and summing over distinct zeros of the Riemann zeta function:

$$
\begin{gathered}
\sum_{0<\gamma_{1} \neq \gamma_{2} \neq \gamma_{3}<T} f\left(\gamma_{1}-\gamma_{2}, \gamma_{1}-\gamma_{3}\right)=\frac{1}{(2 \pi)^{3}} \int_{-T}^{T} \int_{-T}^{T} f\left(v_{1}, v_{2}\right) \\
\times\left[\int_{0}^{T} \log ^{3} \frac{u}{2 \pi} d u+I\left(i v_{1}, i v_{2} ; 0\right)+I\left(0, i v_{1} ;-i v_{2}\right)+I\left(0, i v_{2} ;-i v_{1}\right)\right. \\
+I\left(-i v_{1},-i v_{2} ; 0\right)+I\left(0,-i v_{2} ; i v_{1}\right)+I\left(0,-i v_{1} ; i v_{2}\right) \\
+I_{1}\left(0 ; i v_{2}\right)+I_{1}\left(0 ; i v_{1}\right)+I_{1}\left(-i v_{2} ; i v_{1}\right)+I_{1}\left(-i v_{2} ; 0\right)+I_{1}\left(-i v_{1} ; i v_{2}\right) \\
\left.+I_{1}\left(-i v_{1} ; 0\right)\right] d v_{1} d v_{2}+O\left(T^{\epsilon}\right),
\end{gathered}
$$

where the integrals in $v_{1}$ and $v_{2}$ are to be interpreted as principal value integrals and

$$
\begin{aligned}
I\left(\alpha_{1}, \alpha_{2} ; \beta\right):= & \int_{0}^{T} \frac{\zeta^{\prime}}{\zeta}\left(\frac{1}{2}+i t+\alpha_{1}\right) \frac{\zeta^{\prime}}{\zeta}\left(\frac{1}{2}+i t+\alpha_{2}\right) \frac{\zeta^{\prime}}{\zeta}\left(\frac{1}{2}-i t+\beta\right) d t \\
= & \int_{0}^{T} Q\left(\alpha_{1}+\beta, \alpha_{2}+\beta\right)+\left(\frac{t}{2 \pi}\right)^{-\alpha_{1}-\beta} \zeta\left(1-\alpha_{1}-\beta\right) \zeta\left(1+\alpha_{1}+\beta\right) \\
& \quad \times\left(A\left(\alpha_{1}+\beta\right)\left(\frac{\zeta^{\prime}}{\zeta}\left(1+\alpha_{2}-\alpha_{1}\right)-\frac{\zeta^{\prime}}{\zeta}\left(1+\alpha_{2}+\beta\right)\right)+P\left(\alpha_{1}+\beta, \alpha_{2}+\beta\right)\right) \\
& +\left(\frac{t}{2 \pi}\right)^{-\alpha_{2}-\beta} \zeta\left(1-\alpha_{2}-\beta\right) \zeta\left(1+\alpha_{2}+\beta\right) \\
& \quad \times\left(A\left(\alpha_{2}+\beta\right)\left(\frac{\zeta^{\prime}}{\zeta}\left(1+\alpha_{1}-\alpha_{2}\right)-\frac{\zeta^{\prime}}{\zeta}\left(1+\alpha_{1}+\beta\right)\right)+P\left(\alpha_{2}+\beta, \alpha_{1}+\beta\right)\right) d t \\
& \quad+O\left(T^{1 / 2+\epsilon}\right),
\end{aligned}
$$




$$
\begin{aligned}
I_{1}(\alpha ; \beta):= & \int_{0}^{T} \log \frac{t}{2 \pi} \frac{\zeta^{\prime}}{\zeta}\left(\frac{1}{2}+i t+\alpha\right) \frac{\zeta^{\prime}}{\zeta}\left(\frac{1}{2}-i t+\beta\right) d t \\
= & \int_{0}^{T} \log \frac{t}{2 \pi}\left(\left(\frac{\zeta^{\prime}}{\zeta}\right)^{\prime}(1+\alpha+\beta)\right. \\
& \left.+\left(\frac{t}{2 \pi}\right)^{-\alpha-\beta} \zeta(1+\alpha+\beta) \zeta(1-\alpha-\beta) A(\alpha+\beta)-B(\alpha+\beta)\right) d t \\
& +O\left(T^{1 / 2+\epsilon}\right)
\end{aligned}
$$

and

$$
\begin{gathered}
A(x)=\prod_{p} \frac{\left(1-\frac{1}{p^{1+x}}\right)\left(1-\frac{2}{p}+\frac{1}{p^{1+x}}\right)}{\left(1-\frac{1}{p}\right)^{2}}, \\
B(x)=\sum_{p}\left(\frac{\log p}{p^{1+x}-1}\right)^{2}, \\
Q(x, y)=-\sum_{p} \frac{\log ^{3} p}{p^{2+x+y}\left(1-\frac{1}{p^{1+x}}\right)\left(1-\frac{1}{p^{1+y}}\right)}
\end{gathered}
$$

and

$$
\begin{aligned}
P(x, y)= & \prod_{p} \frac{\left(1-\frac{1}{p^{1+x}}\right)\left(1-\frac{2}{p}+\frac{1}{p^{1+x}}\right)}{\left(1-\frac{1}{p}\right)^{2}} \\
& \times \sum_{p} \frac{\left(1-\frac{1}{p^{x}}\right)\left(1-\frac{1}{p^{x}}-\frac{1}{p^{y}}+\frac{1}{p^{1+y}}\right) \log \frac{1}{p}}{\left(\frac{1}{1-p^{1-x+y}}\right)\left(1-\frac{1}{p^{1+y}}\right)\left(1-\frac{2}{p}+\frac{1}{p^{1+x}}\right) p^{2-x+y}}
\end{aligned}
$$

The expression (2.1) is plotted in Figure 2, with $f\left(v_{1}, v_{2}\right)=\delta\left(v_{1}-x\right) \delta\left(v_{2}-y\right)$. The $\mathrm{x}-$ and $\mathrm{y}$ axes are unscaled, but (2.1) is divided by $T\left(\frac{\log \frac{T}{2 \pi}}{2 \pi}\right)^{3}$. The density plot is light for large values and dark where (2.1) is small. Note the horizontal, vertical and diagonal lines occurring at the Riemann zeros, caused by terms like $\frac{\zeta^{\prime}}{\zeta}(1+i x), \frac{\zeta^{\prime}}{\zeta}(1+i y-i x)$, etc. Numerical computation of (2.1) breaks down near the $\mathrm{x}$ - and $\mathrm{y}$ - axes and on the diagonal because of the principal value integration, so the plot has been set to zero in these regions. The plot could be completed with a more careful expansion of the formula around $x=0, y=0$ and $x=y$, but this would not be particularly edifying. The result would be extremely similar to the random matrix limit shown in Figure 6, The maximum height on the contour plot in Figure 2 is about 0.799. An idea of the height of the plot can be seen in Figure 3. which is a horizontal cross-section of Figure 2 at height 5 on the y-axis.

In Figure 4 the numerical triple correlation, using the first 100000 zeros, is plotted, again scaled as above. The difference between this and Figure 2 is shown in Figure 5 , where the maximum height of the plot is about 0.164. Compare this with the maximum height of Figure 4, which is about 0.923 . The maximum value of the difference plot may seem rather large, as we expect an 


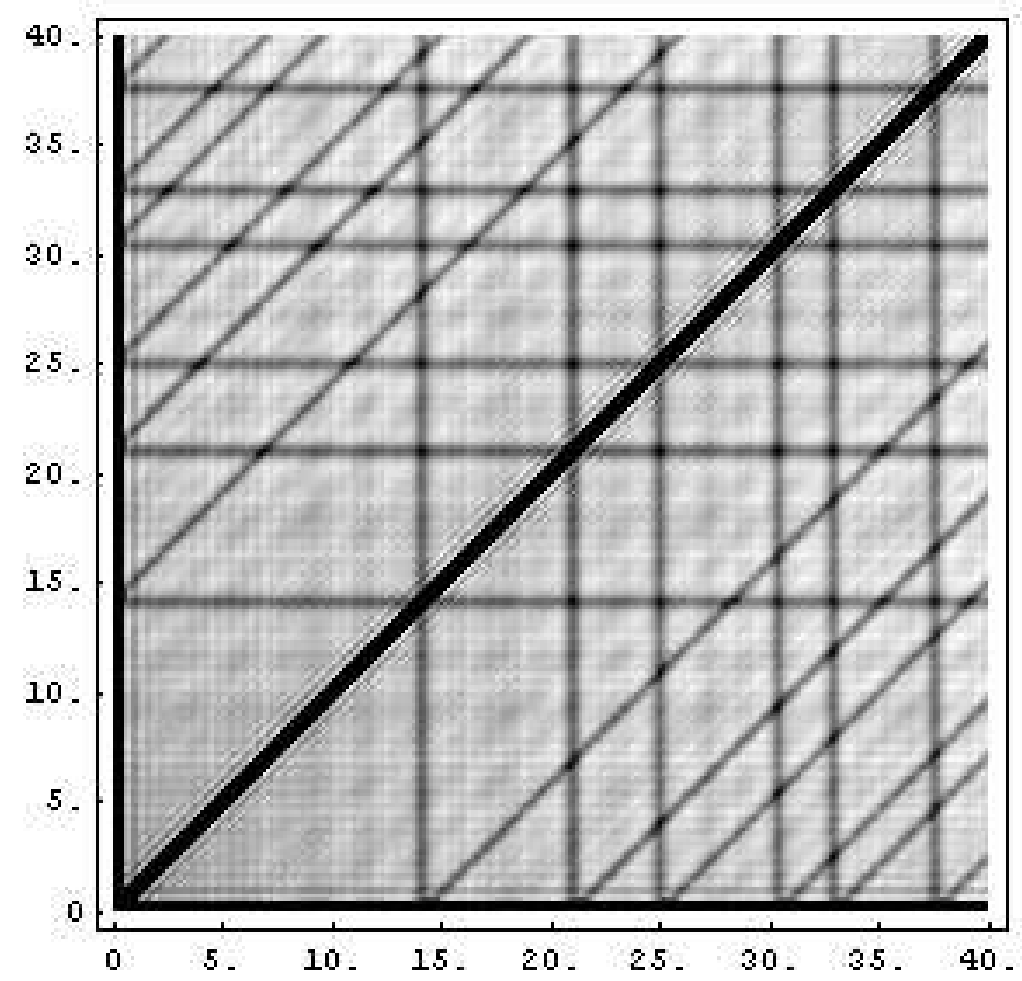

Figure 2. The triple correlation of the Riemann zeros: we plot the quantity in square brackets from (2.1). The $\mathrm{x}$ and $\mathrm{y}$ axes are $v_{1}$ and $v_{2}$ and the density plot is lighter were the function has a higher value and is darker for smaller values. Note the horizontal, vertical and diagonal lines occurring at the Riemann zeros: 14.13, $21.02,25.01, \ldots$

error of $T^{-1 / 2+\epsilon}$, but this is probably due to the relatively small value of $T$ used for these plots. For $T \sim 75000, T^{-1 / 2}$ is around 0.003 , but for $T$ values of this size powers of $\log T$ can make a big difference. The mean value of the points on the difference plot (Figure 5) is -0.00127 , and the standard deviation is about 0.03 , which gives a better idea of the spread of the points. We also note that the mean of the absolute value of Figure 5 is about 0.0257 .

For ease of comparison with Theorem 2.1, we state here the similar random matrix result which is derived in detail in Section 3.2. The identical structure of Theorem 2.1 and Theorem 2.2 is apparent if we recall the equivalence $N=\log \frac{t}{2 \pi}$ (see for example [19]) and reduce (2.8) from three to two variables by performing a simple translation such as $\theta_{2} \rightarrow \theta_{2}+\theta_{1}$ and $\theta_{3} \rightarrow \theta_{3}+\theta_{1}$ and noting from (2.9) and (2.10) that $J(\alpha, \beta ; \gamma)=J(\alpha+t, \beta+t ; \gamma-t)$ and $J(\alpha ; \beta)=J(\alpha+t ; \beta-t)$. In comparing $J(\alpha, \beta ; \gamma)$ with $I(\alpha, \beta ; \gamma)$ and $J(\alpha ; \beta)$ with $I_{1}(\alpha ; \beta), z(x)=\left(1-e^{-x}\right)^{-1}$ plays the part of $\zeta(1+x)$, as is always the case in moment and ratios conjectures. Using the ratios theorem of Conrey, Farmer and Zirnbauer [12, 11] we obtain the following 


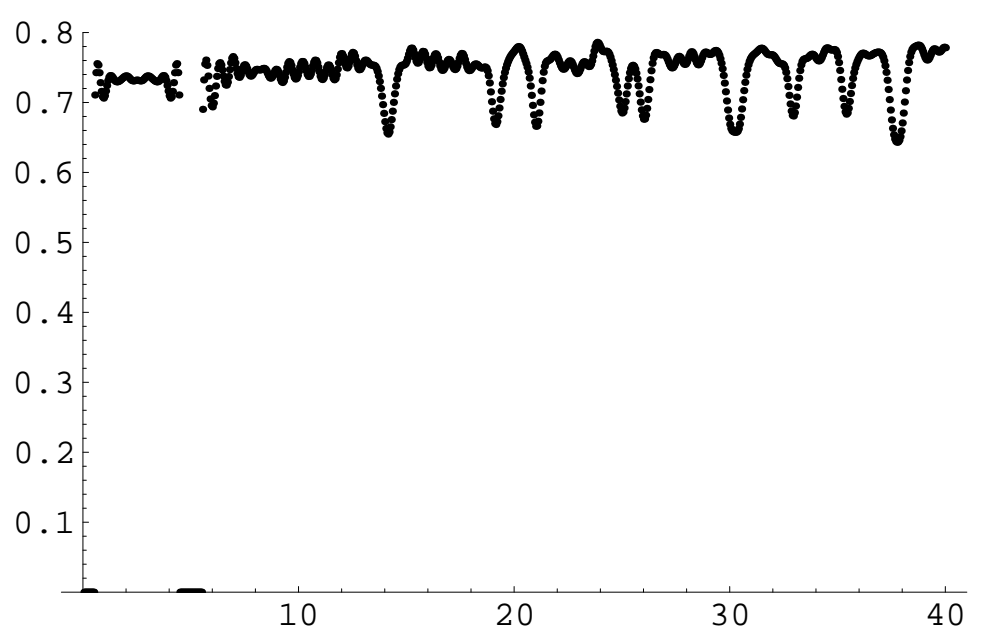

Figure 3. A horizontal cross-section of Figure 2 at height 5 on the y-axis to illustrate the height of that plot.

Theorem 2.2. With the star indicating a sum over distinct eigenvalues we have:

$$
\begin{aligned}
& T_{3}(f):=\int_{U(N)} \sum_{j_{1}, j_{2}, j_{3}}^{*} f\left(\theta_{j_{1}}, \theta_{j_{2}}, \theta_{j_{3}}\right) d X \\
& =\frac{1}{(2 \pi)^{3}} \int_{-\pi}^{\pi} \int_{-\pi}^{\pi} \int_{-\pi}^{\pi}\left(J\left(i \theta_{1}, i \theta_{2} ;-i \theta_{3}\right)\right. \\
& +J\left(i \theta_{1}, i \theta_{3} ;-i \theta_{2}\right)+J\left(i \theta_{2}, i \theta_{3} ;-i \theta_{1}\right)+J\left(-i \theta_{1},-i \theta_{2} ; i \theta_{3}\right) \\
& +J\left(-i \theta_{1},-i \theta_{3} ; i \theta_{2}\right)+J\left(-i \theta_{2},-i \theta_{3} ; i \theta_{1}\right) \\
& +N\left(J\left(-i \theta_{1} ; i \theta_{3}\right)+J\left(-i \theta_{2} ; i \theta_{3}\right)+J\left(-i \theta_{1} ; i \theta_{2}\right)\right. \\
& \left.+J\left(-i \theta_{3} ; i \theta_{2}\right)+J\left(-i \theta_{2} ; i \theta_{1}\right)+J\left(-i \theta_{3} ; i \theta_{1}\right)\right) \\
& \left.+N^{3}\right) f\left(\theta_{1}, \theta_{2}, \theta_{3}\right) d \theta_{1} d \theta_{2} d \theta_{3}
\end{aligned}
$$

where

$$
\begin{aligned}
& J\left(\alpha_{1}, \alpha_{2} ; \beta\right) \\
& :=-e^{-\alpha_{1}-\alpha_{2}-\beta} \int_{U(N)} \frac{\Lambda_{X}^{\prime}}{\Lambda_{X}}\left(e^{-\alpha_{1}}\right) \frac{\Lambda_{X}^{\prime}}{\Lambda_{X}}\left(e^{-\alpha_{2}}\right) \frac{\Lambda_{X^{*}}^{\prime}}{\Lambda_{X^{*}}}\left(e^{-\beta}\right) d X \\
& =e^{-N\left(\alpha_{1}+\beta\right)} z\left(\alpha_{1}+\beta\right) z\left(-\alpha_{1}-\beta\right)\left(\frac{z^{\prime}}{z}\left(\alpha_{2}-\alpha_{1}\right)-\frac{z^{\prime}}{z}\left(\alpha_{2}+\beta\right)\right) \\
& \quad+e^{-N\left(\alpha_{2}+\beta\right)} z\left(\alpha_{2}+\beta\right) z\left(-\alpha_{2}-\beta\right)\left(\frac{z^{\prime}}{z}\left(\alpha_{1}-\alpha_{2}\right)-\frac{z^{\prime}}{z}\left(\alpha_{1}+\beta\right)\right),
\end{aligned}
$$




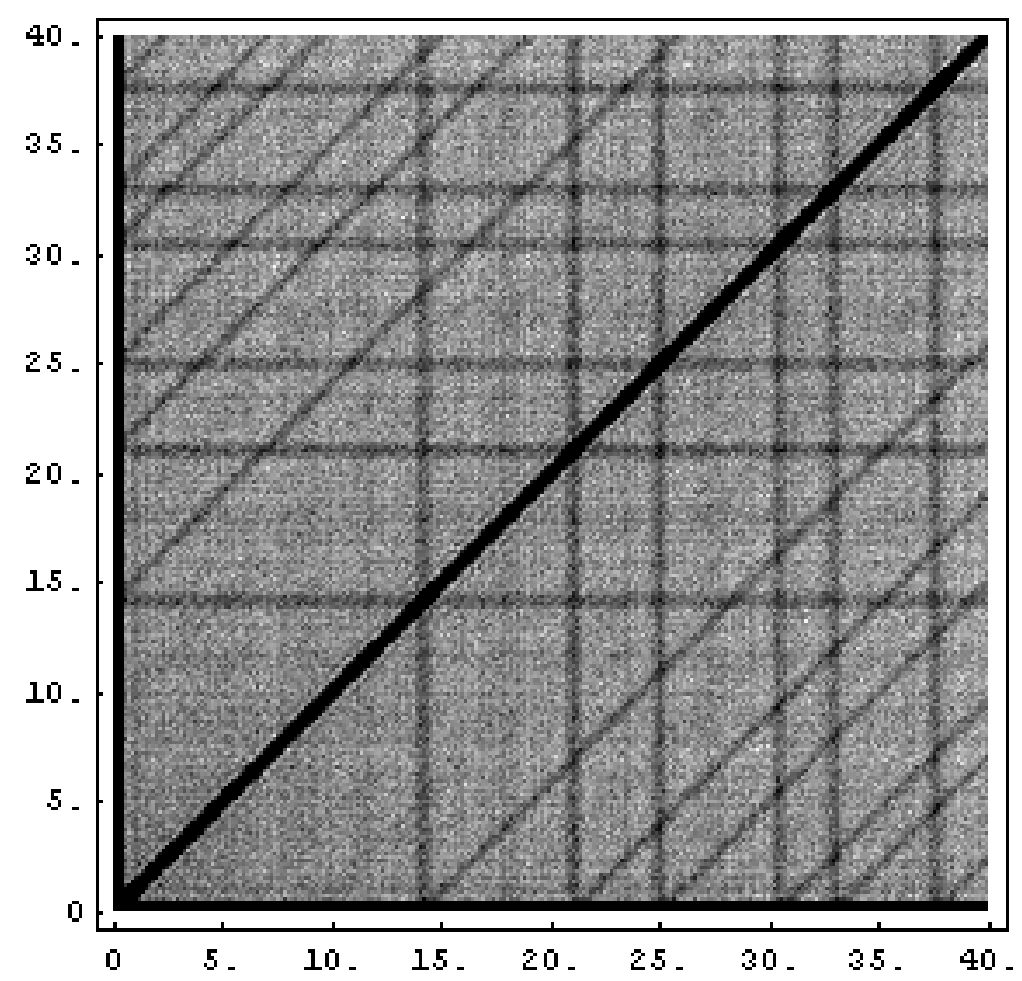

Figure 4. The numerically calculated triple correlation of the Riemann zeros. The distribution of triplets of zeros is depicted by plotting the distance between the first and second zero in the triplet on the horizontal axis against the distance between the first and third zero on the vertical. Higher occurrences appear lighter grey and where no triplets fall the plot is black.

and

$$
\begin{aligned}
J(\alpha ; \beta): & =e^{-\alpha-\beta} \int_{U(N)} \frac{\Lambda_{X}^{\prime}}{\Lambda_{X}}\left(e^{-\alpha}\right) \frac{\Lambda_{X^{*}}^{\prime}}{\Lambda_{X^{*}}}\left(e^{-\beta}\right) d X \\
& =\left(\frac{z^{\prime}}{z}\right)^{\prime}(\alpha+\beta)+e^{-N(\alpha+\beta)} z(\alpha+\beta) z(-\alpha-\beta) .
\end{aligned}
$$

2.2. Moments of the logarithmic derivative of the Riemann zeta function. Calculating correlation functions of the Riemann zeros using the conjectural formulae for averages of ratios of zeta functions proceeds via moments of the logarithmic derivative of the Riemann zeta function:

$$
\begin{aligned}
& I_{r}\left(\alpha_{1}, \ldots, \alpha_{k} ; \beta_{1}, \ldots, \beta_{\ell}\right) \\
& \quad:=\int_{0}^{T} \log ^{r} \frac{t}{2 \pi} \frac{\zeta^{\prime}}{\zeta}\left(\frac{1}{2}+i t+\alpha_{1}\right) \cdots \frac{\zeta^{\prime}}{\zeta}\left(\frac{1}{2}+i t+\alpha_{k}\right) \frac{\zeta^{\prime}}{\zeta}\left(\frac{1}{2}-i t+\beta_{1}\right) \cdots \frac{\zeta^{\prime}}{\zeta}\left(\frac{1}{2}-i t+\beta_{\ell}\right) d t .
\end{aligned}
$$




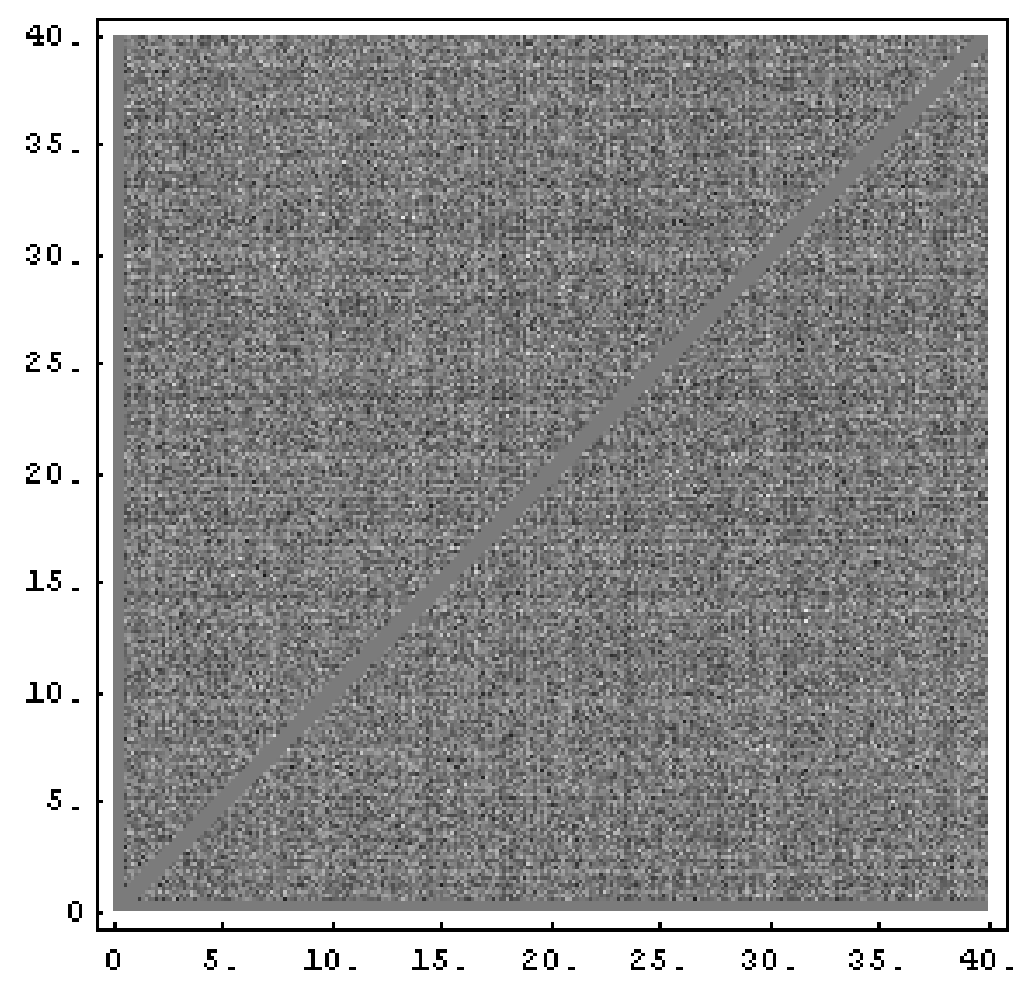

Figure 5. The difference between Figures 4 and 2.

A property of these moments that will be of use to us later is that

$$
I_{r}\left(\alpha_{1}, \ldots, \alpha_{k} ; \beta_{1}, \ldots, \beta_{\ell}\right)=\overline{I_{r}\left(\overline{\beta_{1}}, \ldots, \overline{\beta_{\ell}} ; \overline{\alpha_{1}}, \ldots, \overline{\alpha_{k}}\right)} .
$$

For now the arguments of $I_{r}$ will always have positive real parts. In this case we have an approximate translation invariance: if $\Re\left(\alpha_{i}\right), \Re\left(\beta_{j}\right), \Re\left(\alpha_{i}+\lambda\right), \Re\left(\beta_{j}-\lambda\right)>0$ then

$$
\begin{aligned}
& I_{r}\left(\alpha_{1}+\lambda, \ldots, \alpha_{k}+\lambda ; \beta_{1}-\lambda, \ldots, \beta_{\ell}-\lambda\right) \\
& \quad=I_{r}\left(\alpha_{1}, \ldots, \alpha_{k} ; \beta_{1}, \ldots, \beta_{\ell}\right)+O\left(|\lambda| T^{\epsilon}\right) .
\end{aligned}
$$

This can be seen by a change of variables $t \rightarrow t-i \lambda$ in (2.11) and using $\mathrm{RH}$ to bound $\zeta^{\prime} / \zeta(\sigma+i t)$ by $t^{\epsilon}$.

In particular, to calculate the three-point correlation function, we will need formula (2.2) for $I\left(\alpha_{1}, \alpha_{2} ; \beta\right)$ (here we introduce the convention that $\left.I=I_{0}\right)$.

We will now proceed to derive that formula using the form of the ratios conjecture [1] for three zeta functions over three zeta functions (with the conditions $-\frac{1}{4}<\Re \alpha_{1}, \Re \alpha_{2}, \Re \beta<\frac{1}{4}, \frac{1}{\log T} \ll$ 
$\Re \gamma_{1}, \Re \gamma_{2}, \Re \delta<\frac{1}{4}$ and $\left.\Im \alpha_{1}, \Im \alpha_{2}, \Im \beta, \Im \gamma_{1}, \Im \gamma_{2}, \Im \delta \ll_{\epsilon} T^{1-\epsilon}\right)$ :

$$
\begin{aligned}
& \int_{0}^{T} \frac{\zeta\left(\frac{1}{2}+i t+\alpha_{1}\right) \zeta\left(\frac{1}{2}+i t+\alpha_{2}\right) \zeta\left(\frac{1}{2}-i t+\beta\right)}{\zeta\left(\frac{1}{2}+i t+\gamma_{1}\right) \zeta\left(\frac{1}{2}+i t+\gamma_{2}\right) \zeta\left(\frac{1}{2}-i t+\delta\right)} d t \\
&=\int_{0}^{T} G\left(\alpha_{1}, \alpha_{2}, \beta ; \gamma_{1}, \gamma_{2}, \delta\right)+\left(\frac{t}{2 \pi}\right)^{-\alpha_{1}-\beta} G\left(-\beta, \alpha_{2},-\alpha_{1} ; \gamma_{1}, \gamma_{2}, \delta\right) \\
& \quad+\left(\frac{t}{2 \pi}\right)^{-\alpha_{2}-\beta} G\left(\alpha_{1},-\beta,-\alpha_{2} ; \gamma_{1}, \gamma_{2}, \delta\right) d t+O\left(T^{1 / 2+\epsilon}\right),
\end{aligned}
$$

where the error term is uniform in the specified range of parameters. Here

$$
G\left(\alpha_{1}, \alpha_{2}, \beta ; \gamma_{1}, \gamma_{2}, \delta\right)=Y\left(\alpha_{1}, \alpha_{2}, \beta ; \gamma_{1}, \gamma_{2}, \delta\right) \times A_{\zeta}\left(\alpha_{1}, \alpha_{2}, \beta ; \gamma_{1}, \gamma_{2}, \delta\right)
$$

and

$$
Y\left(\alpha_{1}, \alpha_{2}, \beta ; \gamma_{1}, \gamma_{2}, \delta\right)=\frac{\zeta\left(1+\alpha_{1}+\beta\right) \zeta\left(1+\alpha_{2}+\beta\right) \zeta\left(1+\gamma_{1}+\delta\right) \zeta\left(1+\gamma_{2}+\delta\right)}{\zeta\left(1+\alpha_{1}+\delta\right) \zeta\left(1+\alpha_{2}+\delta\right) \zeta\left(1+\gamma_{1}+\beta\right) \zeta\left(1+\gamma_{2}+\beta\right)}
$$

and

$$
\begin{aligned}
& A_{\zeta}\left(\alpha_{1}, \alpha_{2}, \beta ; \gamma_{1}, \gamma_{2}, \delta\right)= \\
& \quad \prod_{p} \frac{\left(1-\frac{1}{p^{1+\alpha_{1}+\beta}}\right)\left(1-\frac{1}{p^{1+\alpha_{2}+\beta}}\right)\left(1-\frac{1}{p^{1+\gamma_{1}+\delta}}\right)\left(1-\frac{1}{p^{1+\gamma_{2}+\delta}}\right)}{\left(1-\frac{1}{p^{1+\alpha_{1}+\delta}}\right)\left(1-\frac{1}{p^{1+\alpha_{2}+\delta}}\right)\left(1-\frac{1}{p^{1+\gamma_{1}+\beta}}\right)\left(1-\frac{1}{p^{1+\gamma_{2}+\beta}}\right)} \\
& \quad \times \sum_{\substack{m_{1}+m_{2}+h_{1}+h_{2}=n+k \\
m_{1}, m_{2}, h_{1}, h_{2}, n, k \geq 0}} \frac{\mu\left(p^{h_{1}}\right) \mu\left(p^{h_{2}}\right) \mu\left(p^{k}\right)}{p^{m_{1}\left(1 / 2+\alpha_{1}\right)+m_{2}\left(1 / 2+\alpha_{2}\right)+n(1 / 2+\beta)+h_{1}\left(1 / 2+\gamma_{1}\right)+h_{2}\left(1 / 2+\gamma_{2}\right)+k(1 / 2+\delta)}} \\
& =\prod_{p} \frac{\left(1-\frac{1}{p^{1+\gamma_{1}+\delta}}\right)\left(1-\frac{1}{p^{1+\gamma_{2}+\delta}}\right)}{\left(1-\frac{1}{p^{1+\alpha_{1}+\delta}}\right)\left(1-\frac{1}{p^{1+\alpha_{2}+\delta}}\right)\left(1-\frac{1}{p^{1+\gamma_{1}+\beta}}\right)\left(1-\frac{1}{p^{1+\gamma_{2}+\beta}}\right)} \\
& \quad \times\left(1-p^{\beta-\delta}-\frac{1}{p^{1+\gamma_{1}+\beta}}+\frac{1}{p^{1+\gamma_{1}+\delta}}-\frac{1}{p^{1+\gamma_{2}+\beta}}+\frac{1}{p^{1+\gamma_{2}+\delta}}\right. \\
& \left.\quad+\frac{1}{p^{2+\gamma_{1}+\gamma_{2}+2 \beta}}-\frac{1}{p^{2+\gamma_{1}+\gamma_{2}+\beta+\delta}}+p^{\beta-\delta}\left(1-\frac{1}{p^{1+\alpha_{1}+\beta}}\right)\left(1-\frac{1}{p^{1+\alpha_{2}+\beta}}\right)\right) .
\end{aligned}
$$

The main term of (2.14) is analytic in the specified range of parameters; the apparent poles from $Y$ cancel, as can be checked directly, or by writing the three terms as a contour integral as in [11] or Section 2.5 of [10].

Here $\mu(n)$ is the Möbius function and the final expression above reflects the fact that $\mu\left(p^{m}\right)$ is 1 for $m=0$, it is -1 for $m=1$ and zero for any power of $p$ higher than the first. Also note that $A_{\zeta}\left(\alpha_{1}, \alpha_{2}, \beta ; \gamma_{1}, \gamma_{2}, \beta\right)=1$.

We will not go through the reasoning behind this conjecture in full, but a simpler example (one zeta function over one zeta function) can be seen in full detail in [14] and the original reference for the general case is [11]. We will say only that the recipe for arriving at a ratios conjecture involves replacing each zeta function in the numerator with an approximate functional equation, those in the denominator with the Dirichlet series for $\frac{1}{\zeta(s)}$ and then applying a series of rules to discard all the terms in the resulting multiple sums except for those seen above in $A_{\zeta}$. The purpose of $Y$ is to 
factor out the divergent terms in the these sums, leaving $A_{\zeta}$ convergent for small values of $\alpha_{1}, \alpha_{2}$, $\beta, \gamma_{1}, \gamma_{2}$ and $\delta$.

Armed with this ratios conjecture, we want to evaluate

$$
\begin{aligned}
& I\left(\alpha_{1}, \alpha_{2} ; \beta\right) \\
& :=\int_{0}^{T} \frac{\zeta^{\prime}}{\zeta}\left(\frac{1}{2}+i t+\alpha_{1}\right) \frac{\zeta^{\prime}}{\zeta}\left(\frac{1}{2}+i t+\alpha_{2}\right) \frac{\zeta^{\prime}}{\zeta}\left(\frac{1}{2}-i t+\beta\right) d t
\end{aligned}
$$

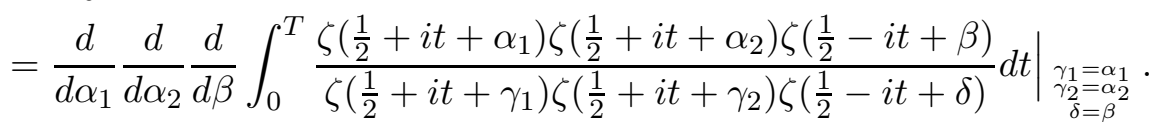

Examining the derivative of the first term in (2.14), we find a great deal of cancellation upon substituting $\gamma_{1}=\alpha_{1}, \gamma_{2}=\alpha_{2}$ and $\delta=\beta$, and the only surviving terms are

$$
\begin{gathered}
\left.\frac{d}{d \alpha_{1}} \frac{d}{d \alpha_{2}} \frac{d}{d \beta} G\left(\alpha_{1}, \alpha_{2}, \beta ; \gamma_{1}, \gamma_{2}, \delta\right)\right|_{\substack{\gamma_{1}=\alpha_{1} \\
\gamma_{2}=\alpha_{2} \\
\delta=\beta}} \\
=\left.\left(\frac{\zeta^{\prime}}{\zeta}\right)^{\prime}\left(1+\alpha_{1}+\beta\right) \frac{d}{d \alpha_{2}} A_{\zeta}\left(\alpha_{1}, \alpha_{2}, \beta ; \alpha_{1}, \gamma_{2}, \beta\right)\right|_{\gamma_{2}=\alpha_{2}} \\
\quad+\left.\left(\frac{\zeta^{\prime}}{\zeta}\right)^{\prime}\left(1+\alpha_{2}+\beta\right) \frac{d}{d \alpha_{1}} A_{\zeta}\left(\alpha_{1}, \alpha_{2}, \beta ; \gamma_{1}, \alpha_{2}, \beta\right)\right|_{\gamma_{1}=\alpha_{1}} \\
\quad+\left.\frac{d}{d \alpha_{1}} \frac{d}{d \alpha_{2}} \frac{d}{d \beta} A_{\zeta}\left(\alpha_{1}, \alpha_{2}, \beta ; \gamma_{1}, \gamma_{2}, \delta\right)\right|_{\substack{\gamma_{1}=\alpha_{1} \\
\gamma_{2}=\alpha_{2} \\
\delta=\beta}} \\
=\left.\frac{d}{d \alpha_{1}} \frac{d}{d \alpha_{2}} \frac{d}{d \beta} A_{\zeta}\left(\alpha_{1}, \alpha_{2}, \beta ; \gamma_{1}, \gamma_{2}, \delta\right)\right|_{\substack{\gamma_{1}=\alpha_{1} \\
\gamma_{2}=\alpha_{2} \\
\delta=\beta}}
\end{gathered}
$$

because $A_{\zeta}\left(\alpha_{1}, \alpha_{2}, \beta ; \gamma_{1}, \gamma_{2}, \beta\right)=1$.

The term

$$
\begin{aligned}
& \frac{d}{d \alpha_{1}} \frac{d}{d \alpha_{2}} \frac{d}{d \beta}\left(\frac{t}{2 \pi}\right)^{-\alpha_{1}-\beta} G\left(-\beta, \alpha_{2},-\alpha_{1} ; \gamma_{1}, \gamma_{2}, \delta\right) \mid \begin{array}{c}
\gamma_{1}=\alpha_{1} \\
\gamma_{2}=\alpha_{2} \\
\delta=\beta
\end{array} \\
& =\frac{d}{d \alpha_{1}} \frac{d}{d \alpha_{2}} \frac{d}{d \beta}\left(\frac{t}{2 \pi}\right)^{-\alpha_{1}-\beta} \frac{\zeta\left(1-\beta-\alpha_{1}\right) \zeta\left(1+\alpha_{2}-\alpha_{1}\right) \zeta\left(1+\gamma_{1}+\delta\right) \zeta\left(1+\gamma_{2}+\delta\right)}{\zeta(1-\beta+\delta) \zeta\left(1+\alpha_{2}+\delta\right) \zeta\left(1+\gamma_{1}-\alpha_{1}\right) \zeta\left(1+\gamma_{2}-\alpha_{1}\right)} \\
& \times A_{\zeta}\left(-\beta, \alpha_{2},-\alpha_{1} ; \gamma_{1}, \gamma_{2}, \delta\right) \mid \begin{array}{c}
\gamma_{1}=\alpha_{1} \\
\gamma_{2}=\alpha_{2} \\
\delta=\beta
\end{array}
\end{aligned}
$$

quickly reduces to

$$
\begin{gathered}
\frac{d}{d \alpha_{2}}\left(\frac{t}{2 \pi}\right)^{-\alpha_{1}-\beta} \frac{\zeta\left(1-\beta-\alpha_{1}\right) \zeta\left(1+\alpha_{2}-\alpha_{1}\right) \zeta\left(1+\alpha_{1}+\beta\right) \zeta\left(1+\gamma_{2}+\beta\right)}{\zeta\left(1+\alpha_{2}+\beta\right) \zeta\left(1+\gamma_{2}-\alpha_{1}\right)} \\
\times\left. A_{\zeta}\left(-\beta, \alpha_{2},-\alpha_{1} ; \alpha_{1}, \gamma_{2}, \beta\right)\right|_{\gamma_{2}=\alpha_{2}}
\end{gathered}
$$

because we see immediately that the factors $\frac{1}{\zeta(1-\beta+\delta)}$ and $\frac{1}{\zeta\left(1+\gamma_{1}-\alpha_{1}\right)}$ cause the entire term to evaluate as zero upon the substitution $\gamma_{1}=\alpha_{1}$ and $\delta=\beta$ unless the $\beta$ and $\alpha_{1}$ derivatives are 
performed on these factors. The final differentiation with respect to $\alpha_{2}$ shows us that (2.20) equals

$$
\begin{aligned}
& \left(\frac{t}{2 \pi}\right)^{-\alpha_{1}-\beta} \zeta\left(1-\beta-\alpha_{1}\right) \zeta\left(1+\beta+\alpha_{1}\right) \\
& \quad \times\left(\left(\frac{\zeta^{\prime}}{\zeta}\left(1+\alpha_{2}-\alpha_{1}\right)-\frac{\zeta^{\prime}}{\zeta}\left(1+\alpha_{2}+\beta\right)\right) A_{\zeta}\left(-\beta, \alpha_{2},-\alpha_{1} ; \alpha_{1}, \alpha_{2}, \beta\right)\right. \\
& \left.\quad+\left.\frac{d}{d \alpha_{2}} A_{\zeta}\left(-\beta, \alpha_{2},-\alpha_{1} ; \alpha_{1}, \gamma_{2}, \beta\right)\right|_{\gamma_{2}=\alpha_{2}}\right) .
\end{aligned}
$$

Similarly,

$$
\begin{aligned}
& \left.\frac{d}{d \alpha_{1}} \frac{d}{d \alpha_{2}} \frac{d}{d \beta}\left(\frac{t}{2 \pi}\right)^{-\alpha_{2}-\beta} G\left(\alpha_{1},-\beta,-\alpha_{2} ; \gamma_{1}, \gamma_{2}, \delta\right)\right|_{\substack{\gamma_{1}=\alpha_{1} \\
\gamma_{2}=\alpha_{2} \\
\delta=\beta}} ^{\substack{\delta_{2} \\
=}}\left(\frac{t}{2 \pi}\right)^{-\alpha_{2}-\beta} \zeta\left(1-\beta-\alpha_{2}\right) \zeta\left(1+\beta+\alpha_{2}\right) \\
& \quad \times\left(\left(\frac{\zeta^{\prime}}{\zeta}\left(1+\alpha_{1}-\alpha_{2}\right)-\frac{\zeta^{\prime}}{\zeta}\left(1+\alpha_{1}+\beta\right)\right) A_{\zeta}\left(\alpha_{1},-\beta,-\alpha_{2} ; \alpha_{1}, \alpha_{2}, \beta\right)\right. \\
& \left.\quad+\left.\frac{d}{d \alpha_{1}} A_{\zeta}\left(\alpha_{1},-\beta,-\alpha_{2} ; \gamma_{1}, \alpha_{2}, \beta\right)\right|_{\gamma_{1}=\alpha_{1}}\right) .
\end{aligned}
$$

Finally, some manipulation of the prime product $A_{\zeta}$ (for which Mathematica is very helpful) shows us (where $A, P$ and $Q$ refer to equations (2.4), (2.7) and (2.6), respectively) that

$$
\begin{aligned}
& A_{\zeta}\left(-\beta, \alpha_{2},-\alpha_{1} ; \alpha_{1}, \alpha_{2}, \beta\right)=A\left(\alpha_{1}+\beta\right) \\
& =\prod_{p} \frac{\left(1-\frac{1}{p^{1+\alpha_{1}+\beta}}\right)\left(1-\frac{2}{p}+\frac{1}{p^{1+\alpha_{1}+\beta}}\right)}{\left(1-\frac{1}{p}\right)^{2}}, \\
& \left.\frac{d}{d \alpha_{2}} A_{\zeta}\left(-\beta, \alpha_{2},-\alpha_{1} ; \alpha_{1}, \gamma_{2}, \beta\right)\right|_{\gamma_{2}=\alpha_{2}}=P\left(\alpha_{1}+\beta, \alpha_{2}+\beta\right) \\
& =\prod_{p} \frac{\left(1-\frac{1}{p^{1+\alpha_{1}+\beta}}\right)\left(1-\frac{2}{p}+\frac{1}{p^{1+\alpha_{1}+\beta}}\right)}{\left(1-\frac{1}{p}\right)^{2}} \\
& \times \sum_{p} \frac{\left(1-\frac{1}{p^{\alpha_{1}+\beta}}\right)\left(1-\frac{1}{p^{\alpha_{1}+\beta}}-\frac{1}{p^{\alpha_{2}+\beta}}+\frac{1}{p^{1+\alpha_{2}+\beta}}\right) \log p}{\left(\frac{1}{p^{1-\alpha_{1}+\alpha_{2}}}-1\right)\left(1-\frac{1}{p^{1+\alpha_{2}+\beta}}\right)\left(1-\frac{2}{p}+\frac{1}{p^{1+\alpha_{1}+\beta}}\right) p^{2-\alpha_{1}+\alpha_{2}}}, \\
& \left.\frac{d}{d \alpha_{1}} A_{\zeta}\left(\alpha_{1},-\beta,-\alpha_{2} ; \gamma_{1}, \alpha_{2}, \beta\right)\right|_{\gamma_{1}=\alpha_{1}}=P\left(\alpha_{2}+\beta, \alpha_{1}+\beta\right), \\
& \left.\frac{d}{d \alpha_{1}} \frac{d}{d \alpha_{2}} \frac{d}{d \beta} A_{\zeta}\left(\alpha_{1}, \alpha_{2}, \beta ; \gamma_{1}, \gamma_{2}, \delta\right)\right|_{\substack{\gamma_{1}=\alpha_{1} \\
\gamma_{2}=\alpha_{2} \\
\delta=\beta}}=Q\left(\alpha_{1}+\beta, \alpha_{2}+\beta\right) \\
& =-\sum_{p} \frac{\log ^{3} p}{p^{2+\alpha_{1}+\alpha_{2}+2 \beta}\left(1-\frac{1}{p^{1+\alpha_{1}+\beta}}\right)\left(1-\frac{1}{p^{1+\alpha_{2}+\beta}}\right)} .
\end{aligned}
$$


Substituting these expressions into (2.19), (2.20) and (2.23), we arrive at (2.2) as expected.

The other version of (2.11) that we need is $I_{1}(\alpha ; \beta)$, as given in (2.3). This calculation has in fact already been carried out in [14] using the two zeta functions over two zeta functions ratios conjecture in a manner very similar to the three-over-three calculation above. That is [12, 11], with $-\frac{1}{4}<\Re \alpha, \Re \beta<\frac{1}{4}, \frac{1}{\log T} \ll \Re \gamma, \Re \delta<\frac{1}{4}$ and $\Im \alpha, \Im \beta, \Im \gamma, \Im \delta \ll_{\epsilon} T^{1-\epsilon}$ (for every $\epsilon>0$ ), the ratios conjecture states

$$
\begin{aligned}
\int_{0}^{T} & \frac{\zeta(s+\alpha) \zeta(1-s+\beta)}{\zeta(s+\gamma) \zeta(1-s+\delta)} d t \\
\quad= & \int_{0}^{T}\left(\frac{\zeta(1+\alpha+\beta) \zeta(1+\gamma+\delta)}{\zeta(1+\alpha+\delta) \zeta(1+\beta+\gamma)} A_{\zeta}(\alpha, \beta ; \gamma, \delta)\right. \\
& \left.\quad+\left(\frac{t}{2 \pi}\right)^{-\alpha-\beta} \frac{\zeta(1-\alpha-\beta) \zeta(1+\gamma+\delta)}{\zeta(1-\beta+\delta) \zeta(1-\alpha+\gamma)} A_{\zeta}(-\beta,-\alpha ; \gamma, \delta)\right) d t+O\left(T^{1 / 2+\epsilon}\right)
\end{aligned}
$$

where

$$
A_{\zeta}(\alpha, \beta ; \gamma, \delta)=\prod_{p} \frac{\left(1-\frac{1}{p^{1+\gamma+\delta}}\right)\left(1-\frac{1}{p^{1+\beta+\gamma}}-\frac{1}{p^{1+\alpha+\delta}}+\frac{1}{p^{1+\gamma+\delta}}\right)}{\left(1-\frac{1}{p^{1+\beta+\gamma}}\right)\left(1-\frac{1}{p^{1+\alpha+\delta}}\right)}
$$

This implies that

$$
\begin{aligned}
& \int_{0}^{T} \frac{\zeta^{\prime}}{\zeta}(s+\alpha) \frac{\zeta^{\prime}}{\zeta}(1-s+\beta) d t=\int_{0}^{T}\left(\left(\frac{\zeta^{\prime}}{\zeta}\right)^{\prime}(1+\alpha+\beta)+\right. \\
& \left(\frac{t}{2 \pi}\right)^{-\alpha-\beta} \zeta(1+\alpha+\beta) \zeta(1-\alpha-\beta) \prod_{p} \frac{\left(1-\frac{1}{p^{1+\alpha+\beta}}\right)\left(1-\frac{2}{p}+\frac{1}{p^{1+\alpha+\beta}}\right)}{\left(1-\frac{1}{p}\right)^{2}} \\
& \left.\quad-\sum_{p}\left(\frac{\log p}{\left(p^{1+\alpha+\beta}-1\right)}\right)^{2}\right) d t+O\left(T^{1 / 2+\epsilon}\right)
\end{aligned}
$$

provided that $\frac{1}{\log T} \ll \Re \alpha, \Re \beta<\frac{1}{4}$. The ratios conjecture recipe can incorporate the $\log \frac{t}{2 \pi}$ factor in $I_{1}(\alpha ; \beta)$ without any alteration, giving (2.3).

2.3. Triple correlation as a contour integral. We start with the a triple sum over zeros of the Riemann zeta function, where the zeros do not have to be distinct. Using Cauchy's residue theorem, the triple sum can be written as a triple contour integral where each contour is a rectangle enclosing the zeros $1 / 2+i \gamma$ (assuming the Riemann Hypothesis) with heights $0 \leq \gamma<T$ :

$$
\begin{array}{rl}
\sum_{0 \leq \gamma_{1}, \gamma_{2}, \gamma_{3}<T} & g\left(\gamma_{1}, \gamma_{2}, \gamma_{3}\right) \\
= & \frac{1}{(2 \pi i)^{3}} \oint \oint \oint g(-i(x-1 / 2),-i(y-1 / 2),-i(z-1 / 2)) \frac{\zeta^{\prime}}{\zeta}(x) \frac{\zeta^{\prime}}{\zeta}(y) \frac{\zeta^{\prime}}{\zeta}(z) d x d y d z .
\end{array}
$$

In investigating the 3-point correlation of the Riemann zeros, we are interested in the relative spacing between triples of zeros, so we can assume that the test function is translation invariant. 
Thus we define a function $f$ satisfying the conditions

$$
\begin{aligned}
& f(x, y) \text { is holomorphic for }|\Im x|<2,|\Im y|<2 \\
& \text { and that } f(x, y) \ll 1 /\left(1+|x|^{2}+|y|^{2}\right) \text { as }|x| \text { or }|y| \rightarrow \infty .
\end{aligned}
$$

Thus we have

$$
\begin{aligned}
\sum_{0 \leq \gamma_{1}, \gamma_{2}, \gamma_{3}<T} f\left(\gamma_{1}-\gamma_{2}, \gamma_{1}-\gamma_{3}\right)= & \frac{1}{(2 \pi i)^{3}} \oint \oint \oint \frac{\zeta^{\prime}}{\zeta}\left(z_{1}\right) \frac{\zeta^{\prime}}{\zeta}\left(z_{2}\right) \frac{\zeta^{\prime}}{\zeta}\left(z_{3}\right) \\
& \times f\left(-i\left(z_{1}-z_{2}\right),-i\left(z_{1}-z_{3}\right)\right) d z_{1} d z_{2} d z_{3},
\end{aligned}
$$

where the contours are rectangles with corners at the points $(a, 0),(a, i T),(1-b, i T)$ and $(1-b, 0)$ with $1 / 2<a, b<1$. We distinguish $a$ and $b$ only for ease of following the manipulations in the following calculations.

The horizontal portions of the contour of integration can be chosen so that the integral along them is negligible, so we concentrate on the vertical sides of the contours. This makes (2.1) the sum of eight integrals $J_{1}, \ldots, J_{8}$ which will be met one by one below (a subscript $a$ on an integral indicates integration from $(a, 0)$ to $(a, i T))$.

First we have

$$
\begin{aligned}
J_{1}= & \frac{1}{(2 \pi i)^{3}} \int_{a} \int_{a} \int_{a} \frac{\zeta^{\prime}}{\zeta}\left(z_{1}\right) \frac{\zeta^{\prime}}{\zeta}\left(z_{2}\right) \frac{\zeta^{\prime}}{\zeta}\left(z_{3}\right) \\
& \quad \times f\left(-i\left(z_{1}-z_{2}\right),-i\left(z_{1}-z_{3}\right)\right) d z_{1} d z_{2} d z_{3} \\
= & O\left(T^{\epsilon}\right) .
\end{aligned}
$$

The final line is true because all three contours can be moved to the right (assuming the Riemann Hypothesis) where $\frac{\zeta^{\prime}}{\zeta}$ converges and can be integrated term by term (the pole at 1 doesn't contribute more than a constant).

Next we examine

$$
\begin{aligned}
J_{2}:=-\frac{1}{(2 \pi i)^{3}} \int_{a} \int_{a} \int_{1-b} \frac{\zeta^{\prime}}{\zeta}\left(z_{1}\right) \frac{\zeta^{\prime}}{\zeta}\left(z_{2}\right) \frac{\zeta^{\prime}}{\zeta}\left(z_{3}\right) \\
\quad \times f\left(-i\left(z_{1}-z_{2}\right),-i\left(z_{1}-z_{3}\right)\right) d z_{1} d z_{2} d z_{3} .
\end{aligned}
$$

We use the functional equation

$$
\frac{\zeta^{\prime}}{\zeta}(s)=\frac{\chi^{\prime}}{\chi}(s)-\frac{\zeta^{\prime}}{\zeta}(1-s)
$$

and obtain

$$
\begin{gathered}
J_{2}=-\frac{1}{(2 \pi i)^{3}} \int_{a} \int_{a} \int_{1-b}\left(\frac{\chi^{\prime}}{\chi}\left(z_{1}\right)-\frac{\zeta^{\prime}}{\zeta}\left(1-z_{1}\right)\right) \frac{\zeta^{\prime}}{\zeta}\left(z_{2}\right) \frac{\zeta^{\prime}}{\zeta}\left(z_{3}\right) \\
\times f\left(-i\left(z_{1}-z_{2}\right),-i\left(z_{1}-z_{3}\right)\right) d z_{1} d z_{2} d z_{3} .
\end{gathered}
$$

For the term with $\frac{\chi^{\prime}}{\chi}\left(z_{1}\right)$ we can shift the integrals to the right, as we did with $J_{1}$, and so see that the contribution is only $O\left(T^{\epsilon}\right)$. A similar manoeuver cannot be done with the integral containing 
the three $\frac{\zeta^{\prime}}{\zeta}$, however, because the $z_{1}$ integral is to the left of the critical line. So,

$$
\begin{aligned}
J_{2}=\frac{1}{(2 \pi)^{3}} & \int_{0}^{T} \int_{0}^{T} \int_{0}^{T} \frac{\zeta^{\prime}}{\zeta}\left(b-i t_{1}\right) \frac{\zeta^{\prime}}{\zeta}\left(a+i t_{2}\right) \frac{\zeta^{\prime}}{\zeta}\left(a+i t_{3}\right) \\
& \times f\left(t_{1}-t_{2}-i(1-b-a), t_{1}-t_{3}-i(1-b-a)\right) d t_{1} d t_{2} d t_{3}+O\left(T^{\epsilon}\right) .
\end{aligned}
$$

Now, letting $v_{1}=t_{2}-t_{1}$ and $v_{2}=t_{3}-t_{1}$, we have

$$
\begin{aligned}
J_{2}= & \frac{1}{(2 \pi)^{3}} \int_{0}^{T} \int_{-t_{1}}^{T-t_{1}} \int_{-t_{1}}^{T-t_{1}} \frac{\zeta^{\prime}}{\zeta}\left(b-i t_{1}\right) \frac{\zeta^{\prime}}{\zeta}\left(a+i\left(v_{1}+t_{1}\right)\right) \frac{\zeta^{\prime}}{\zeta}\left(a+i\left(v_{2}+t_{1}\right)\right) \\
& \times f\left(-v_{1}-i(1-b-a),-v_{2}-i(1-b-a)\right) d v_{1} d v_{2} d t_{1}+O\left(T^{\epsilon}\right) \\
= & \frac{1}{(2 \pi)^{3}} \int_{-T}^{T} \int_{-T}^{T} f\left(-v_{1}-i(1-b-a),-v_{2}-i(1-b-a)\right) \\
& \times \int_{0}^{T} \frac{\zeta^{\prime}}{\zeta}\left(b-i t_{1}\right) \frac{\zeta^{\prime}}{\zeta}\left(a+i\left(v_{1}+t_{1}\right)\right) \frac{\zeta^{\prime}}{\zeta}\left(a+i\left(v_{2}+t_{1}\right)\right) d t_{1} d v_{1} d v_{2}+O\left(T^{\epsilon}\right) .
\end{aligned}
$$

In this last line we have switched the order of integration of $t_{1}$ with $v_{1}$ and $v_{2}$. The range of the innermost integral should really be from $\max \left\{0,-v_{1},-v_{2}\right\}$ to $\min \left\{T, T-v_{1}, T-v_{2}\right\}$. However, since we are assuming that $f(x, y)$ decays fast, see (2.32), it is not hard to show that extending the interval to $(0, T)$ as we have done above, and will do again in the following integrals $J_{3}$ to $J_{8}$, incurs only an error that is a power of $\log T$ and so can be incorporated into the error term $O\left(T^{\epsilon}\right)$.

Now all that is left is to tidy up the expression for $J_{2}$, so we shift the contours of integration off the real axis and apply the definition of $I\left(\alpha_{1}, \alpha_{2} ; \beta\right)$ from (2.11) with $r=0$ :

$$
\begin{aligned}
J_{2}= & \frac{1}{(2 \pi)^{3}} \int_{-T-i(1-b-a)}^{T-i(1-b-a)} \int_{-T-i(1-b-a)}^{T-i(1-b-a)} f\left(v_{1}, v_{2}\right) \\
& \quad \times \int_{0}^{T} \frac{\zeta^{\prime}}{\zeta}\left(b-i t_{1}\right) \frac{\zeta^{\prime}}{\zeta}\left(1+i t_{1}-b-i v_{1}\right) \frac{\zeta^{\prime}}{\zeta}\left(1+i t_{1}-b-i v_{2}\right) d t_{1} d v_{1} d v_{2}+O\left(T^{\epsilon}\right) \\
= & \frac{1}{(2 \pi)^{3}} \int_{-T-i(1-b-a)}^{T-i(1-b-a)} \int_{-T-i(1-b-a)}^{T-i(1-b-a)} f\left(v_{1}, v_{2}\right) I\left(-i v_{1},-i v_{2} ; 0\right) d v_{1} d v_{2}+O\left(T^{\epsilon}\right) .
\end{aligned}
$$

To simplify the last line we have used (2.13).

We use exactly the same sequence of manipulations to obtain similar expressions for $J_{3}$ and $J_{4}$ :

$$
\begin{aligned}
J_{3}:= & -\frac{1}{(2 \pi i)^{3}} \int_{a} \int_{1-b} \int_{a} \frac{\zeta^{\prime}}{\zeta}\left(z_{1}\right) \frac{\zeta^{\prime}}{\zeta}\left(z_{2}\right) \frac{\zeta^{\prime}}{\zeta}\left(z_{3}\right) \\
& \quad \times f\left(-i\left(z_{1}-z_{2}\right),-i\left(z_{1}-z_{3}\right)\right) d z_{1} d z_{2} d z_{3} \\
= & \frac{1}{(2 \pi)^{3}} \int_{-T}^{T} \int_{-T+i(1-b-a)}^{T+i(1-b-a)} f\left(v_{1}, v_{2}\right) I\left(0,-i v_{2} ; i v_{1}\right) d v_{1} d v_{2}+O\left(T^{\epsilon}\right)
\end{aligned}
$$


and

$$
\begin{aligned}
J_{4}:= & -\frac{1}{(2 \pi i)^{3}} \int_{1-b} \int_{a} \int_{a} \frac{\zeta^{\prime}}{\zeta}\left(z_{1}\right) \frac{\zeta^{\prime}}{\zeta}\left(z_{2}\right) \frac{\zeta^{\prime}}{\zeta}\left(z_{3}\right) \\
& \quad \times f\left(-i\left(z_{1}-z_{2}\right),-i\left(z_{1}-z_{3}\right)\right) d z_{1} d z_{2} d z_{3} \\
= & \frac{1}{(2 \pi)^{3}} \int_{-T+i(1-b-a)}^{T+i(1-b-a)} \int_{-T}^{T} f\left(v_{1}, v_{2}\right) I\left(0,-i v_{1} ; i v_{2}\right) d v_{1} d v_{2}+O\left(T^{\epsilon}\right) .
\end{aligned}
$$

The integral $J_{5}$ throws up something slightly different. We start off in an identical manner, replacing $\frac{\zeta^{\prime}}{\zeta}\left(z_{2}\right)$ and $\frac{\zeta^{\prime}}{\zeta}\left(z_{3}\right)$ by their functional equation (2.36). This results in four terms, one of which contributes no more than $O\left(T^{\epsilon}\right)$. We are left with three terms:

$$
\begin{aligned}
& J_{5}:=\frac{1}{(2 \pi i)^{3}} \int_{1-b} \int_{1-b} \int_{a} \frac{\zeta^{\prime}}{\zeta}\left(z_{1}\right) \frac{\zeta^{\prime}}{\zeta}\left(z_{2}\right) \frac{\zeta^{\prime}}{\zeta}\left(z_{3}\right) \\
& \times f\left(-i\left(z_{1}-z_{2}\right),-i\left(z_{1}-z_{3}\right)\right) d z_{1} d z_{2} d z_{3} \\
& =\frac{1}{(2 \pi)^{3}} \int_{0}^{T} \int_{0}^{T} \int_{0}^{T}\left(\frac{\zeta^{\prime}}{\zeta}\left(a+i t_{1}\right) \frac{\zeta^{\prime}}{\zeta}\left(b-i t_{2}\right) \frac{\zeta^{\prime}}{\zeta}\left(b-i t_{3}\right)\right. \\
& -\frac{\chi^{\prime}}{\chi}\left(1-b+i t_{2}\right) \frac{\zeta^{\prime}}{\zeta}\left(a+i t_{1}\right) \frac{\zeta^{\prime}}{\zeta}\left(b-i t_{3}\right) \\
& \left.-\frac{\chi^{\prime}}{\chi}\left(1-b+i t_{3}\right) \frac{\zeta^{\prime}}{\zeta}\left(a+i t_{1}\right) \frac{\zeta^{\prime}}{\zeta}\left(b-i t_{2}\right)\right) \\
& \times f\left(t_{1}-t_{2}+i(1-a-b), t_{1}-t_{3}+i(1-a-b)\right) d t_{1} d t_{2} d t_{3}+O\left(T^{\epsilon}\right) \\
& =\frac{1}{(2 \pi)^{3}} \int_{0}^{T} \int_{-t_{1}}^{T-t_{1}} \int_{-t_{1}}^{T-t_{1}}\left(\frac{\zeta^{\prime}}{\zeta}\left(a+i t_{1}\right) \frac{\zeta^{\prime}}{\zeta}\left(b-i\left(v_{1}+t_{1}\right)\right) \frac{\zeta^{\prime}}{\zeta}\left(b-i\left(v_{2}+t_{1}\right)\right)\right. \\
& -\frac{\chi^{\prime}}{\chi}\left(1-b+i\left(v_{1}+t_{1}\right)\right) \frac{\zeta^{\prime}}{\zeta}\left(a+i t_{1}\right) \frac{\zeta^{\prime}}{\zeta}\left(b-i\left(v_{2}+t_{1}\right)\right) \\
& \left.-\frac{\chi^{\prime}}{\chi}\left(1-b+i\left(v_{2}+t_{1}\right)\right) \frac{\zeta^{\prime}}{\zeta}\left(a+i t_{1}\right) \frac{\zeta^{\prime}}{\zeta}\left(b-i\left(v_{1}+t_{1}\right)\right)\right) \\
& \times f\left(-v_{1}+i(1-a-b),-v_{2}+i(1-a-b)\right) d v_{1} d v_{2} d t_{1}+O\left(T^{\epsilon}\right),
\end{aligned}
$$

where in the last line we have made our usual change of variables $v_{1}=t_{2}-t_{1}$ and $v_{2}=t_{3}-t_{1}$.

We now note the asymptotic for $\frac{\chi^{\prime}}{\chi}$ :

$$
\frac{\chi^{\prime}}{\chi}(1 / 2+i t)=-\log \frac{|t|}{2 \pi}\left(1+O\left(\frac{1}{|t|}\right)\right) .
$$


Since $f$ is very small when $v_{1}$ or $v_{2}$ are large, we replace $\frac{\chi^{\prime}}{\chi}\left(1-b+i\left(v_{j}+z_{1}\right)\right)$ with $-\log \frac{z_{1}}{2 \pi}$ and obtain

$$
\begin{aligned}
J_{5}=\frac{1}{(2 \pi)^{3}} \int_{-T}^{T} \int_{-T}^{T} f\left(-v_{1}+i(1-a-b),-v_{2}+i(1-a-b)\right) \\
\times \int_{0}^{T}\left(\frac{\zeta^{\prime}}{\zeta}\left(a+i t_{1}\right) \frac{\zeta^{\prime}}{\zeta}\left(b-i\left(v_{1}+t_{1}\right)\right) \frac{\zeta^{\prime}}{\zeta}\left(b-i\left(v_{2}+t_{1}\right)\right)\right. \\
+\log \frac{t_{1}}{2 \pi} \frac{\zeta^{\prime}}{\zeta}\left(a+i t_{1}\right) \frac{\zeta^{\prime}}{\zeta}\left(b-i\left(v_{2}+t_{1}\right)\right) \\
\left.+\log \frac{t_{1}}{2 \pi} \frac{\zeta^{\prime}}{\zeta}\left(a+i t_{1}\right) \frac{\zeta^{\prime}}{\zeta}\left(b-i\left(v_{1}+t_{1}\right)\right)\right) d t_{1} d v_{1} d v_{2}+O\left(T^{\epsilon}\right),
\end{aligned}
$$

where we have extended the range of $t_{1}$, after exchanging the order of integration, by the same argument as for $J_{2}$.

Now we can write $J_{5}$ as a double integral along contours running just below the real axis, in a similar form to $J_{2}, J_{3}$ and $J_{4}$ above, but we introduce $I_{1}(\alpha ; \beta)$ (see (2.11) and (2.3)) to arrive at

$$
\begin{array}{r}
J_{5}=\frac{1}{(2 \pi)^{3}} \int_{-T+i(1-a-b)}^{T+i(1-a-b)} \int_{-T+i(1-a-b)}^{T+i(1-a-b)} f\left(v_{1}, v_{2}\right)\left(I\left(0 ; i v_{1}, i v_{2}\right)\right. \\
\left.+I_{1}\left(0 ; i v_{2}\right)+I_{1}\left(0 ; i v_{1}\right)\right) d v_{1} d v_{2}+O\left(T^{\epsilon}\right) .
\end{array}
$$

Proceeding exactly as for $J_{5}$, we obtain similar expressions for $J_{6}$ and $J_{7}$ :

$$
\begin{aligned}
J_{6}:= & \frac{1}{(2 \pi i)^{3}} \int_{1-b} \int_{a} \int_{1-b} \frac{\zeta^{\prime}}{\zeta}\left(z_{1}\right) \frac{\zeta^{\prime}}{\zeta}\left(z_{2}\right) \frac{\zeta^{\prime}}{\zeta}\left(z_{3}\right) \\
& \times f\left(-i\left(z_{1}-z_{2}\right),-i\left(z_{1}-z_{3}\right)\right) d z_{1} d z_{2} d z_{3} \\
= & \frac{1}{(2 \pi)^{3}} \int_{-T}^{T} \int_{-T-i(1-a-b)}^{T-i(1-a-b)} f\left(v_{1}, v_{2}\right)\left(I\left(-i v_{1} ; 0, i v_{2}\right)\right. \\
& \left.\quad+I_{1}\left(-i v_{1} ; i v_{2}\right)+I_{1}\left(-i v_{1} ; 0\right)\right) d v_{1} d v_{2}+O\left(T^{\epsilon}\right)
\end{aligned}
$$

and

$$
\begin{aligned}
& J_{7}:= \frac{1}{(2 \pi i)^{3}} \int_{a} \int_{1-b} \int_{1-b} \frac{\zeta^{\prime}}{\zeta}\left(z_{1}\right) \frac{\zeta^{\prime}}{\zeta}\left(z_{2}\right) \frac{\zeta^{\prime}}{\zeta}\left(z_{3}\right) \\
& \quad \times f\left(-i\left(z_{1}-z_{2}\right),-i\left(z_{1}-z_{3}\right)\right) d z_{1} d z_{2} d z_{3} \\
&=\frac{1}{(2 \pi)^{3}} \int_{-T-i(1-a-b)}^{T-i(1-a-b)} \int_{-T}^{T} f\left(v_{1}, v_{2}\right)\left(I\left(-i v_{2} ; 0, i v_{1}\right)\right. \\
&\left.\quad+I_{1}\left(-i v_{2} ; i v_{1}\right)+I_{1}\left(-i v_{2} ; 0\right)\right) d v_{1} d v_{2}+O\left(T^{\epsilon}\right) .
\end{aligned}
$$


We are just left with the integral

$$
\begin{aligned}
J_{8}:=-\frac{1}{(2 \pi i)^{3}} \int_{1-b} \int_{1-b} \int_{1-b} \frac{\zeta^{\prime}}{\zeta}\left(z_{1}\right) \frac{\zeta^{\prime}}{\zeta}\left(z_{2}\right) \frac{\zeta^{\prime}}{\zeta}\left(z_{3}\right) \\
\quad \times f\left(-i\left(z_{1}-z_{2}\right),-i\left(z_{1}-z_{3}\right)\right) d z_{1} d z_{2} d z_{3}
\end{aligned}
$$

to evaluate. Once each $\frac{\zeta^{\prime}}{\zeta}(z)$ is replaced by its functional equation (2.36), any term with at least one $\frac{\zeta^{\prime}}{\zeta}(1-z)$ in it can be shown to be size just $O\left(T^{\epsilon}\right)$ by shifting the contour far to the left. This leaves us with

$$
\begin{array}{r}
J_{8}=-\frac{1}{(2 \pi)^{3}} \int_{0}^{T} \int_{0}^{T} \int_{0}^{T} \frac{\chi^{\prime}}{\chi}\left(\frac{1}{2}+i t_{1}\right) \frac{\chi^{\prime}}{\chi}\left(\frac{1}{2}+i t_{2}\right) \frac{\chi^{\prime}}{\chi}\left(\frac{1}{2}+i t_{3}\right) \\
\times f\left(t_{1}-t_{2}, t_{1}-t_{3}\right) d t_{1} d t_{2} d t_{3}+O\left(T^{\epsilon}\right) .
\end{array}
$$

Here the contours have all been moved onto the half-line as there are no longer zeros of zeta functions to avoid. We replace $t_{2}-t_{1}$ with $v_{1}$ and replace $t_{3}-t_{1}$ with $v_{2}$ and substitute $-\log \frac{t_{1}}{2 \pi}$ for each $\frac{\chi^{\prime}}{\chi}$ factor with the help of (2.44) , as we did in the discussion of $J_{5}$, and so obtain

$$
J_{8}=\frac{1}{(2 \pi)^{3}} \int_{-T}^{T} \int_{-T}^{T} f\left(v_{1}, v_{2}\right) \int_{0}^{T} \log ^{3} \frac{t}{2 \pi} d t d v_{1} d v_{2}+O\left(T^{\epsilon}\right)
$$

The sum of integrals $J_{1}, \ldots, J_{8}$ gives us (see (2.53) ) the expression for

$$
\sum_{0 \leq \gamma_{1}, \gamma_{2}, \gamma_{3}<T} f\left(\gamma_{1}-\gamma_{2}, \gamma_{1}-\gamma_{3}\right) .
$$

Such a triple sum over zeros necessarily contains terms where two zeros are identical. These are essentially two-point correlations, rather than three-point statistics. Similarly, terms where all three zeros are identical are just one-point statistics. To remove these lower-order correlations, one looks at the sum

$$
\sum_{0<\gamma_{1} \neq \gamma_{2} \neq \gamma_{3}<T} f\left(\gamma_{1}-\gamma_{2}, \gamma_{1}-\gamma_{3}\right)
$$

instead. As will be shown in the next section, simply rewriting as an integral on the real line each of the integrals above that occurs with a contour on $(-T-i(1-a-b), T-i(1-a-b))$ or $(-T+i(1-a-b), T+i(1-a-b))$ gives us the purely triple correlation, and so we obtain the final result (2.1). The contributions from the poles that we meet as the contours are shifted to the real axes are the source of the two- and one-point correlation terms. 
2.4. Contributions from lower-order correlations. Collecting together the results from the previous section we have

$$
\begin{aligned}
& \sum_{0 \leq \gamma_{1}, \gamma_{2}, \gamma_{3}<T} f\left(\gamma_{1}-\gamma_{2}, \gamma_{1}-\gamma_{3}\right)=\frac{1}{(2 \pi)^{3}}\left(\int_{-T}^{T} \int_{-T}^{T} f\left(v_{1}, v_{2}\right) \int_{0}^{T} \log ^{3} \frac{t}{2 \pi} d t d v_{1} d v_{2}\right. \\
& +\int_{-T+i(1-a-b)}^{T+i(1-a-b)} \int_{-T+i(1-a-b)}^{T+i(1-a-b)} f\left(v_{1}, v_{2}\right)\left(I\left(0 ; i v_{1}, i v_{2}\right)\right. \\
& \left.\quad+I_{1}\left(0 ; i v_{2}\right)+I_{1}\left(0 ; i v_{1}\right)\right) d v_{1} d v_{2} \\
& +\int_{-T}^{T} \int_{-T-i(1-a-b)}^{T-i(1-a-b)} f\left(v_{1}, v_{2}\right)\left(I\left(-i v_{1} ; 0, i v_{2}\right)\right. \\
& \left.\quad+I_{1}\left(-i v_{1} ; i v_{2}\right)+I_{1}\left(-i v_{1} ; 0\right)\right) d v_{1} d v_{2} \\
& +\int_{-T-i(1-a-b)}^{T-i(1-a-b)} \int_{-T}^{T} f\left(v_{1}, v_{2}\right)\left(I\left(-i v_{2} ; 0, i v_{1}\right)\right. \\
& \left.\quad+I_{1}\left(-i v_{2} ; i v_{1}\right)+I_{1}\left(-i v_{2} ; 0\right)\right) d v_{1} d v_{2} \\
& +\int_{-T-i(1-b-a)}^{T-i(1-b-a)} \int_{-T-i(1-b-a)}^{T-i(1-b-a)} f\left(v_{1}, v_{2}\right) I\left(-i v_{1},-i v_{2} ; 0\right) d v_{1} d v_{2} \\
& +\int_{-T}^{T} \int_{-T+i(1-b-a)}^{T+i(1-b-a)} f\left(v_{1}, v_{2}\right) I\left(0,-i v_{2} ; i v_{1}\right) d v_{1} d v_{2} \\
& \left.+\int_{-T+i(1-b-a)}^{T+i(1-b-a)} \int_{-T}^{T} f\left(v_{1}, v_{2}\right) I\left(0,-i v_{1} ; i v_{2}\right) d v_{1} d v_{2}\right)+O\left(T^{\epsilon}\right) .
\end{aligned}
$$

The goal of this section is to move all the contours of integration above onto the real axis, evaluating the contributions from the poles encountered during this process. The resulting integrals along the real axis will then be computable, for the purposes of Figure ???? for example, as principal value integrals. Elegantly, the terms resulting from the residue at the various poles will yield the contribution to (2.53) from lower-order correlations between Riemann zeros.

Consider, as an example of the method, the term (coming from $J_{5}$ )

$$
\int_{-T+i(1-a-b)}^{T+i(1-a-b)} \int_{-T+i(1-a-b)}^{T+i(1-a-b)} f\left(v_{1}, v_{2}\right) I\left(i v_{1}, i v_{2} ; 0\right) d v_{1} d v_{2}
$$

from (2.53). (Here we have switched the order of the arguments of $I$ using (2.12).) Treating the integrand first as a function of $v_{2}$ alone (leaving $v_{1}$ fixed and non-zero), we see that the $v_{2}$ contour lies below the real axis (since $a>1 / 2$ and $b>1 / 2$ ) and there is a pole at $v_{2}=0$, so shifting the contour onto the axis results in a principal value integral in $v_{2}$ plus $i \pi$ times the residue of that pole. Using the definition of the sums and products over primes found in (2.4) to (2.7), expanding 
around $v_{2}=0$ gives us

$$
\begin{aligned}
Q\left(i v_{1}, i v_{2}\right) & =O(1) \\
A\left(i v_{2}\right) & =1+O\left(v_{2}^{2}\right) \\
P\left(i v_{2}, i v_{1}\right) & =i v_{2} \sum_{p} \frac{\log ^{2} p}{\left(p^{1+i v_{1}}-1\right)^{2}}+O\left(v_{2}^{2}\right) \\
P\left(i v_{1}, i v_{2}\right) & =O(1) .
\end{aligned}
$$

These, in conjunction with the poles of $I\left(i v_{1}, i v_{2} ; 0\right)$ at $v_{2}=0$ resulting from $\zeta\left(1-i v_{2}\right) \zeta\left(1+i v_{2}\right)$ and $\frac{\zeta^{\prime}}{\zeta}\left(1+i v_{2}\right)$, allows us to express (2.54) as

$$
\begin{gathered}
\int_{-T}^{T} \int_{-T+i(1-a-b)}^{T+i(1-a-b)} f\left(v_{1}, v_{2}\right) I\left(i v_{1}, i v_{2} ; 0\right) d v_{1} d v_{2} \\
+\pi \int_{-T+i(1-a-b)}^{T+i(1-a-b)} \int_{0}^{T}\left(\left(\frac{\zeta^{\prime}}{\zeta}\right)^{\prime}\left(1+i v_{1}\right)+\left(\frac{t}{2 \pi}\right)^{-i v_{1}} \zeta\left(1+i v_{1}\right) \zeta\left(1-i v_{1}\right) A\left(i v_{1}\right)\right. \\
\left.\quad-\sum_{p} \frac{\log ^{2} p}{\left(p^{1+i v_{1}}-1\right)^{2}}\right) f\left(v_{1}, 0\right) d t d v_{1}
\end{gathered}
$$

where the $v_{2}$ integral is understood as a principal value integral.

Now we move the $v_{1}$ integral onto the real axis. In this case also we encounter a pole at $v_{1}=0$. The first integral in (2.59) yields a residue contribution essentially identical to that from the $v_{2}$ pole treated above, and the second integral has a pole at $v_{1}=0$ with residue $-i \log \frac{t}{2 \pi}$. We take $i \pi$ times the contributions from these poles and the end result is

$$
\begin{aligned}
\int_{-T}^{T} \int_{-T}^{T} f\left(v_{1}, v_{2}\right) I\left(i v_{1}, i v_{2} ; 0\right) d v_{1} d v_{2} \\
+\pi \int_{-T}^{T} f\left(v_{1}, 0\right) \int_{0}^{T}\left(\left(\frac{\zeta^{\prime}}{\zeta}\right)^{\prime}\left(1+i v_{1}\right)+\left(\frac{t}{2 \pi}\right)^{-i v_{1}} \zeta\left(1+i v_{1}\right) \zeta\left(1-i v_{1}\right) A\left(i v_{1}\right)\right. \\
\left.\quad-\sum_{p} \frac{\log ^{2} p}{\left(p^{1+i v_{1}}-1\right)^{2}}\right) d t d v_{1} \\
+\pi \int_{-T}^{T} f\left(0, v_{2}\right) \int_{0}^{T}\left(\left(\frac{\zeta^{\prime}}{\zeta}\right)^{\prime}\left(1+i v_{2}\right)+\left(\frac{t}{2 \pi}\right)^{-i v_{2}} \zeta\left(1+i v_{2}\right) \zeta\left(1-i v_{2}\right) A\left(i v_{2}\right)\right. \\
\left.\quad-\sum_{p} \frac{\log ^{2} p}{\left(p^{1+i v_{2}}-1\right)^{2}}\right) d t d v_{2} \\
+\pi^{2} \int_{0}^{T} f(0,0) \log \frac{t}{2 \pi} d t .
\end{aligned}
$$

We note that the final term comprises information on the one-point correlation function, whereas the second and third integrals in (2.60) will form a contribution to the two-point correlation function, as will be discussed at the end of this section. 
In the meantime we will perform sample calculations on three other terms from (2.53), leaving the remainder of the terms to the reader, since they all follow a similar pattern.

We consider now

$$
\int_{-T+i(1-a-b)}^{T+i(1-a-b)} \int_{-T+i(1-a-b)}^{T+i(1-a-b)} f\left(v_{1}, v_{2}\right) I_{1}\left(0 ; i v_{2}\right) d v_{1} d v_{2}
$$

Examining expression (2.3) for $I_{1}\left(0 ; i v_{2}\right)$, we see that the $\left(\frac{\zeta^{\prime}}{\zeta}\right)^{\prime}$ term has a second order pole at $v_{2}=0$ with residue zero, the term containing $\zeta\left(1+i v_{2}\right) \zeta\left(1-i v_{2}\right)$ has residue

$$
\int_{0}^{T}-i \log ^{2} \frac{t}{2 \pi} d t
$$

and $B\left(0, v_{2}\right)$ is analytic near $v_{2}=0$. The integrand is analytic in $v_{1}$, so moving that contour onto the real axis does not incur any polar contribution. Thus (2.61) equals

$$
\int_{-T}^{T} \int_{-T}^{T} f\left(v_{1}, v_{2}\right) I_{1}\left(0 ; v_{2}\right) d v_{1} d v_{2}+\pi \int_{-T}^{T} f\left(v_{1}, 0\right) \int_{0}^{T} \log ^{2} \frac{t}{2 \pi} d t d v_{1}
$$

The next term that deserves consideration is

$$
\int_{-T-i(1-a-b)}^{T-i(1-a-b)} \int_{-T}^{T} f\left(v_{1}, v_{2}\right) I\left(i v_{1}, 0 ;-i v_{2}\right) d v_{1} d v_{2}
$$

where again we have used (2.12) to exchange the arguments of $I$ preceding the semicolon with those following it. We note that in these integrals where two consecutive principal value calculations are made the order of the integrals cannot be exchanged after the first principal value integral is obtained, so we always address the principal values starting with the outermost integral and working inwards.

An inspection of (2.2) reveals that $I\left(i v_{1}, 0 ;-i v_{2}\right)$ is not singular at $v_{1}=0$ (the poles of two $\frac{\zeta^{\prime}}{\zeta}$ terms cancel), but as we move the $v_{2}$ contour onto the real axis we encounter extra difficulties when $v_{1}=0$, so we will start by temporarily shifting the $v_{1}$ contour so that it runs just below the real axis; we choose below rather than above the axis so as to avoid the pole at $v_{1}=v_{2}$.

Now that the $v_{1}$ contour does not pass through zero, we can move the $v_{2}$ contour onto the real axis and pick up exactly the same polar contribution as when evaluating (2.54). (An extra minus sign in the residue compensates for the fact that this time we need it multiplied by $-i \pi$ since we are half-circling the pole in the clockwise direction due to the original contour passing above the real axis.) Thus, for some $\epsilon>0$, (2.64) is

$$
\begin{aligned}
\int_{-T}^{T} \int_{-T-i \epsilon}^{T-i \epsilon} & f\left(v_{1}, v_{2}\right) I\left(i v_{1}, 0 ;-i v_{2}\right) d v_{1} d v_{2} \\
+\pi & \int_{-T-i \epsilon}^{T-i \epsilon} \int_{0}^{T}\left(\left(\frac{\zeta^{\prime}}{\zeta}\right)^{\prime}\left(1+i v_{1}\right)+\left(\frac{t}{2 \pi}\right)^{-i v_{1}} \zeta\left(1+i v_{1}\right) \zeta\left(1-i v_{1}\right) A\left(i v_{1}\right)\right. \\
& \left.-\sum_{p} \frac{\log ^{2} p}{\left(p^{1+i v_{1}}-1\right)^{2}}\right) f\left(v_{1}, 0\right) d t d v_{1},
\end{aligned}
$$

where the $v_{2}$ integral is understood as a principal value integral. 
As we move the $v_{1}$ contour back to the real axis, we encounter a pole of the first integral above at $v_{1}=v_{2}$ (the apparent pole at $v_{1}=0$ in fact cancels), and a pole of the second integral at $v_{1}=0$ with residue $-i \log \frac{t}{2 \pi}$, as before. The final result is that (2.64) equals

$$
\begin{aligned}
\int_{-T}^{T} \int_{-T}^{T} f\left(v_{1}, v_{2}\right) I\left(i v_{1}, 0 ;-i v_{2}\right) d v_{1} d v_{2} \\
+\pi \int_{-T}^{T} f\left(v_{1}, 0\right) \int_{0}^{T}\left(\left(\frac{\zeta^{\prime}}{\zeta}\right)^{\prime}\left(1+i v_{1}\right)+\left(\frac{t}{2 \pi}\right)^{-i v_{1}} \zeta\left(1+i v_{1}\right) \zeta\left(1-i v_{1}\right) A\left(i v_{1}\right)\right. \\
\left.\quad-\sum_{p} \frac{\log ^{2} p}{\left(p^{1+i v_{1}}-1\right)^{2}}\right) d t d v_{1} \\
+\pi \int_{-T}^{T} f\left(v_{2}, v_{2}\right) \int_{0}^{T}\left(\left(\frac{\zeta^{\prime}}{\zeta}\right)^{\prime}\left(1-i v_{2}\right)+\left(\frac{t}{2 \pi}\right)^{i v_{2}} \zeta\left(1+i v_{2}\right) \zeta\left(1-i v_{2}\right) A\left(-i v_{2}\right)\right. \\
\left.\quad-\sum_{p} \frac{\log ^{2} p}{\left(p^{1-i v_{2}}-1\right)^{2}}\right) d t d v_{2} \\
+\pi^{2} \int_{0}^{T} f(0,0) \log \frac{t}{2 \pi} d t .
\end{aligned}
$$

The last term we will consider (all the others follow in an identical manner to one of those discussed) is

$$
\int_{-T}^{T} \int_{-T-i(1-a-b)}^{T-i(1-a-b)} f\left(v_{1}, v_{2}\right) I\left(i v_{2}, 0 ;-i v_{1}\right) d v_{1} d v_{2}
$$

where as usual we have used (2.12) to exchange the arguments of $I$ preceding the semicolon with those following it. We write this as

$$
\lim _{\epsilon \rightarrow 0^{+}} \int_{\substack{[-T, T] \\\left|v_{2}\right|>\epsilon}} \int_{-T-i(1-a-b)}^{T-i(1-a-b)} f\left(v_{1}, v_{2}\right) I\left(i v_{2}, 0 ;-i v_{1}\right) d v_{1} d v_{2}
$$

This has not changed a thing because the outer integral is perfectly well-behaved at $v_{2}=0$, but it means that as we move the inner integral onto the real axis we avoid the complications that arise if $v_{2}=0$. 
The inner integral has poles at $v_{1}=0$ and $v_{1}=v_{2}$ with the usual and now-familiar residues, so (2.67) equals

$$
\begin{aligned}
\int_{-T}^{T} \int_{-T}^{T} f\left(v_{1}, v_{2}\right) I\left(i v_{2}, 0 ;-i v_{1}\right) d v_{1} d v_{2} \\
+\pi \int_{-T}^{T} f\left(0, v_{2}\right) \int_{0}^{T}\left(\left(\frac{\zeta^{\prime}}{\zeta}\right)^{\prime}\left(1+i v_{2}\right)+\left(\frac{t}{2 \pi}\right)^{-i v_{2}} \zeta\left(1+i v_{2}\right) \zeta\left(1-i v_{2}\right) A\left(i v_{2}\right)\right. \\
\left.\quad-\sum_{p} \frac{\log ^{2} p}{\left(p^{1+i v_{2}}-1\right)^{2}}\right) d t d v_{2} \\
+\pi \int_{-T}^{T} f\left(v_{2}, v_{2}\right) \int_{0}^{T}\left(\left(\frac{\zeta^{\prime}}{\zeta}\right)^{\prime}\left(1-i v_{2}\right)+\left(\frac{t}{2 \pi}\right)^{i v_{2}} \zeta\left(1+i v_{2}\right) \zeta\left(1-i v_{2}\right) A\left(-i v_{2}\right)\right. \\
\left.\quad-\sum_{p} \frac{\log ^{2} p}{\left(p^{1-i v_{2}}-1\right)^{2}}\right) d t d v_{2},
\end{aligned}
$$

where the integrals in $v_{1}$ and $v_{2}$ are to be interpreted as principal value integrals.

All of the other terms in (2.53) can be handled in exactly the same way as the one of the four treated here. The complete result is

$$
\begin{gathered}
\sum_{0 \leq \gamma_{1}, \gamma_{2}, \gamma_{3}<T} f\left(\gamma_{1}-\gamma_{2}, \gamma_{1}-\gamma_{3}\right)=\frac{1}{(2 \pi)^{3}} \int_{-T}^{T} \int_{-T}^{T} f\left(v_{1}, v_{2}\right) \\
\times\left(\int_{0}^{T} \log ^{3} \frac{u}{2 \pi} d u+I\left(i v_{1}, i v_{2} ; 0\right)+I\left(0, i v_{1} ;-i v_{2}\right)+I\left(0, i v_{2} ;-i v_{1}\right)\right. \\
\quad+I\left(-i v_{1},-i v_{2} ; 0\right)+I\left(0,-i v_{2} ; i v_{1}\right)+I\left(0,-i v_{1} ; i v_{2}\right) \\
+I_{1}\left(0 ; i v_{2}\right)+I_{1}\left(0 ; i v_{1}\right)+I_{1}\left(-i v_{2} ; i v_{1}\right)+I_{1}\left(-i v_{2} ; 0\right)+I_{1}\left(-i v_{1} ; i v_{2}\right) \\
\left.\quad+I_{1}\left(-i v_{1} ; 0\right)\right) d v_{1} d v_{2} \\
+\frac{1}{(2 \pi)^{2}}\left(\int_{-T}^{T} f\left(0, v_{2}\right) s\left(v_{2}\right) d v_{2}+\int_{-T}^{T} f\left(v_{1}, 0\right) s\left(v_{1}\right) d v_{1}+\int_{-T}^{T} f\left(v_{1}, v_{1}\right) s\left(-v_{1}\right) d v_{1}\right. \\
\left.+\int_{-T}^{T} f\left(0, v_{2}\right) s\left(-v_{2}\right) d v_{2}+\int_{-T}^{T} f\left(v_{1}, 0\right) s\left(-v_{1}\right) d v_{1}+\int_{-T}^{T} f\left(v_{1}, v_{1}\right) s\left(v_{1}\right) d v_{1}\right) \\
+\frac{1}{2 \pi} \int_{0}^{T} f(0,0) \log \frac{t}{2 \pi} d t,
\end{gathered}
$$

where

$$
\begin{gathered}
s(x)=\int_{0}^{T} \frac{1}{2} \log ^{2} \frac{t}{2 \pi}+\left(\frac{\zeta^{\prime}}{\zeta}\right)^{\prime}(1+i x)+\left(\frac{t}{2 \pi}\right)^{-i x} \zeta(1+i x) \zeta(1-i x) A(i x) \\
-\sum_{p} \frac{\log ^{2} p}{\left(p^{1+i x}-1\right)^{2}} d t .
\end{gathered}
$$


Note that some terms above can be combined if we include the natural assumption that $f(x, y)=$ $f(-x,-y)$. All the integrals on the interval $(-T, T)$ should be considered as principal value integrals near any poles at the origin or at $v_{1}=v_{2}$.

Now we want to identify the terms in the last three lines of (2.70) as lower order correlations. To do this, note that we can rewrite the triple sum over zeta zeros as

$$
\begin{aligned}
& \quad \sum_{0<\gamma_{1}, \gamma_{2}, \gamma_{3}<T} f\left(\gamma_{1}-\gamma_{2}, \gamma_{1}-\gamma_{3}\right)=\sum_{0<\gamma_{1} \neq \gamma_{2} \neq \gamma_{3}<T} f\left(\gamma_{1}-\gamma_{2}, \gamma_{1}-\gamma_{3}\right) \\
& \quad+\sum_{0<\gamma_{1} \neq \gamma_{2}<T} f\left(\gamma_{1}-\gamma_{2}, 0\right)+\sum_{0<\gamma_{1} \neq \gamma_{3}<T} f\left(0, \gamma_{1}-\gamma_{3}\right)+\sum_{0<\gamma_{1} \neq \gamma_{2}<T} f\left(\gamma_{1}-\gamma_{2}, \gamma_{1}-\gamma_{2}\right) \\
& \quad+\sum_{0<\gamma_{1}<T} f(0,0) .
\end{aligned}
$$

The standard result on the density of the zeros of the Riemann zeta function gives,

$$
\sum_{0<\gamma_{1}<T} 1=\frac{T}{2 \pi} \log \frac{T}{2 \pi}-\frac{T}{2 \pi}+O(1),
$$

for large $T$, and an expression for the two-point correlation, derived from the ratios conjecture, is given in [14]:

$$
\begin{aligned}
\sum_{\gamma \neq \gamma^{\prime} \leq T} f\left(\gamma-\gamma^{\prime}\right)=\frac{1}{(2 \pi)^{2}} \int_{-T}^{T} \int_{0}^{T} f(r)\left(\log ^{2} \frac{t}{2 \pi}+\left(\frac{\zeta^{\prime}}{\zeta}\right)^{\prime}(1+i r)\right. \\
+\left(\frac{t}{2 \pi}\right)^{-i r} \zeta(1-i r) \zeta(1+i r) A(i r)-B(i r)+\left(\frac{\zeta^{\prime}}{\zeta}\right)^{\prime}(1-i r) \\
\left.+\left(\frac{t}{2 \pi}\right)^{i r} \zeta(1-i r) \zeta(1+i r) A(-i r)-B(-i r)\right) d t d r+O\left(T^{1 / 2+\epsilon}\right)
\end{aligned}
$$

where $f(z)$ is holomorphic throughout the strip $|\Im z|<2$, is real on the real line and satisfies $f(x) \ll 1 /\left(1+x^{2}\right)$ as $x \rightarrow \infty$. In addition, the integral is to be regarded as a principal value near $r=0$,

$$
A(\eta)=\prod_{p} \frac{\left(1-\frac{1}{p^{1+\eta}}\right)\left(1-\frac{2}{p}+\frac{1}{p^{1+\eta}}\right)}{\left(1-\frac{1}{p}\right)^{2}}
$$

and

$$
B(\eta)=\sum_{p} \frac{\log ^{2} p}{\left(p^{1+\eta}-1\right)^{2}}
$$

We see immediately from (2.73), (2.74) and (2.72) that $\sum_{0<\gamma_{1} \neq \gamma_{2} \neq \gamma_{3}<T} f\left(\gamma_{1}-\gamma_{2}, \gamma_{1}-\gamma_{3}\right)$ is given by the first five lines of (2.70), and this is the result presented in Theorem 2.1 . 
2.5. Retrieving the asymptotic result. We want to confirm that our formula for the triple correlation of the Riemann zeros (2.1) tends to the limit (plotted in Figure 6)

$$
\begin{aligned}
\lim _{T \rightarrow \infty} \frac{1}{\frac{T}{2 \pi} \log \frac{T}{2 \pi}} \sum_{0<\gamma_{1} \neq \gamma_{2} \neq \gamma_{3}<T} f\left(\frac{\log \frac{T}{2 \pi}}{2 \pi}\left(\gamma_{1}-\gamma_{2}\right), \frac{\log \frac{T}{2 \pi}}{2 \pi}\left(\gamma_{1}-\gamma_{3}\right)\right) \\
=\int_{-\infty}^{\infty} \int_{-\infty}^{\infty} f\left(v_{1}, v_{2}\right)\left|\begin{array}{ccc}
1 & S\left(v_{1}\right) & S\left(v_{2}\right) \\
S\left(v_{1}\right) & 1 & S\left(v_{1}-v_{2}\right) \\
S\left(v_{2}\right) & S\left(v_{1}-v_{2}\right)
\end{array}\right| d v_{1} d v_{2} \\
=\int_{-\infty}^{\infty} \int_{-\infty}^{\infty} f\left(v_{1}, v_{2}\right)\left(1-\frac{\sin ^{2}\left(\pi\left(v_{1}-v_{2}\right)\right)}{\pi^{2}\left(v_{1}-v_{2}\right)^{2}}-\frac{\sin ^{2}\left(\pi v_{1}\right)}{\pi^{2} v_{1}^{2}}-\frac{\sin ^{2}\left(\pi v_{2}\right)}{\pi^{2} v_{2}^{2}}\right. \\
\left.+2 \frac{\sin \left(\pi v_{1}\right)}{\pi v_{1}} \frac{\sin \left(\pi v_{2}\right)}{\pi v_{2}} \frac{\sin \left(\pi\left(v_{1}-v_{2}\right)\right)}{\pi\left(v_{1}-v_{2}\right)}\right) d v_{1} d v_{2} .
\end{aligned}
$$

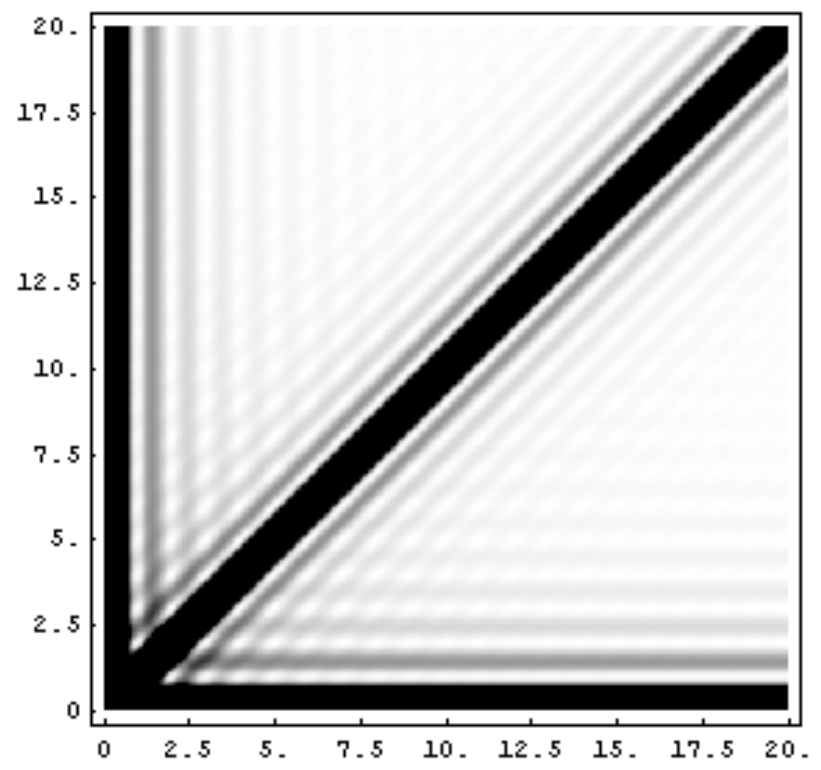

FiguRE 6. The triple correlation of eigenvalues of random matrices from $U(N)$ in the limit as $N \rightarrow \infty$. That is, we have plotted the $3 \times 3$ determinant from the second line of (2.77). Note that to compare this picture to Figures 2 and 4 the axes of those figures would need to be scaled by the mean density of zeros, $\left(\log \frac{T}{2 \pi}\right) /(2 \pi)$. 
Using (2.1), with $L=\log \frac{T}{2 \pi}$, we scale the variables in the test function by $\frac{L}{2 \pi}$, and make a change of variables $v \rightarrow \frac{2 \pi v}{L}$ in the $v_{1}$ and $v_{2}$ integrals:

$$
\begin{gathered}
\sum_{0<\gamma_{1} \neq \gamma_{2} \neq \gamma_{3}<T} f\left(\frac{L}{2 \pi}\left(\gamma_{1}-\gamma_{2}\right), \frac{L}{2 \pi}\left(\gamma_{1}-\gamma_{3}\right)\right) \\
=\frac{1}{L^{2} 2 \pi} \int_{-\frac{T L}{2 \pi}}^{\frac{T L}{2 \pi}} \int_{-\frac{T L}{2 \pi}}^{\frac{T L}{2 \pi}} f\left(v_{1}, v_{2}\right) \\
\times\left(\int_{0}^{T} \log ^{3} \frac{u}{2 \pi} d u+I\left(\frac{2 \pi i v_{1}}{L}, \frac{2 \pi i v_{2}}{L} ; 0\right)+I\left(0, \frac{2 \pi i v_{1}}{L} ;-\frac{2 \pi i v_{2}}{L}\right)+I\left(0, \frac{2 \pi i v_{2}}{L} ;-\frac{2 \pi i v_{1}}{L}\right)\right. \\
\quad+I\left(-\frac{2 \pi i v_{1}}{L},-\frac{2 \pi i v_{2}}{L} ; 0\right)+I\left(0,-\frac{2 \pi i v_{2}}{L} ; \frac{2 \pi i v_{1}}{L}\right)+I\left(0,-\frac{2 \pi i v_{1}}{L} ; \frac{2 \pi i v_{2}}{L}\right) \\
+I_{1}\left(0 ; \frac{2 \pi i v_{2}}{L}\right)+I_{1}\left(0 ; \frac{2 \pi i v_{1}}{L}\right)+I_{1}\left(-\frac{2 \pi i v_{2}}{L} ; \frac{2 \pi i v_{1}}{L}\right)+I_{1}\left(-\frac{2 \pi i v_{2}}{L} ; 0\right) \\
\left.\quad+I_{1}\left(-\frac{2 \pi i v_{1}}{L} ; \frac{2 \pi i v_{2}}{L}\right)+I_{1}\left(-\frac{2 \pi i v_{1}}{L} ; 0\right)\right) d v_{1} d v_{2}+O\left(T^{\epsilon}\right)
\end{gathered}
$$

Starting with the first term of (2.78), we see that asymptotically for large $T$,

$$
\begin{gathered}
\frac{1}{L^{2} 2 \pi} \int_{-\frac{T L}{2 \pi}}^{\frac{T L}{2 \pi}} \int_{-\frac{T L}{2 \pi}}^{\frac{T L}{2 \pi}} f\left(v_{1}, v_{2}\right) \int_{0}^{T} \log ^{3} \frac{t}{2 \pi} d t d v_{1} d v_{2} \\
\sim \frac{T}{2 \pi} \log \frac{T}{2 \pi} \int_{-\infty}^{\infty} \int_{-\infty}^{\infty} f\left(v_{1}, v_{2}\right) d v_{1} d v_{2} .
\end{gathered}
$$

A little more work is needed for the other terms of (2.78). Next we consider

$$
\frac{1}{L^{2} 2 \pi} \int_{-\frac{T L}{2 \pi}}^{\frac{T L}{2 \pi}} \int_{-\frac{T L}{2 \pi}}^{\frac{T L}{2 \pi}} f\left(v_{1}, v_{2}\right) I\left(\frac{2 \pi i v_{1}}{L}, \frac{2 \pi i v_{2}}{L} ; 0\right) d v_{1} d v_{2} .
$$

Using the definition of $I\left(\alpha_{1}, \alpha_{2} ; \beta\right)$ in (2.2), we will be a little imprecise and discard any terms that will not ultimately contribute to the leading-order $T \log \frac{T}{2 \pi}$ result. We thus keep only terms with third-order poles as $T \rightarrow \infty$, and the leading-order contribution to (2.80) is contained in

$$
\begin{aligned}
& \frac{1}{L^{2} 2 \pi} \int_{-}^{\frac{T L}{2 \pi}} \int_{-\frac{T L}{2 \pi}}^{\frac{T L}{2 \pi}} f\left(v_{1}, v_{2}\right) \\
& \quad \times \int_{0}^{T}\left(\left(\frac{t}{2 \pi}\right)^{-\frac{2 \pi i v_{1}}{L}} \zeta\left(1-\frac{2 \pi i v_{1}}{L}\right) \zeta\left(1+\frac{2 \pi i v_{1}}{L}\right) A\left(\frac{2 \pi i v_{1}}{L}\right) \frac{\zeta^{\prime}}{\zeta}\left(1+\frac{2 \pi i v_{2}}{L}-\frac{2 \pi i v_{1}}{L}\right)\right. \\
&-\left(\frac{t}{2 \pi}\right)^{-\frac{2 \pi i v_{1}}{L}} \zeta\left(1-\frac{2 \pi i v_{1}}{L}\right) \zeta\left(1+\frac{2 \pi i v_{1}}{L}\right) A\left(\frac{2 \pi i v_{1}}{L}\right) \frac{\zeta^{\prime}}{\zeta}\left(1+\frac{2 \pi i v_{2}}{L}\right) \\
&+\left(\frac{t}{2 \pi}\right)^{-\frac{2 \pi i v_{2}}{L}} \zeta\left(1-\frac{2 \pi i v_{2}}{L}\right) \zeta\left(1+\frac{2 \pi i v_{2}}{L}\right) A\left(\frac{2 \pi i v_{2}}{L}\right) \frac{\zeta^{\prime}}{\zeta}\left(1+\frac{2 \pi i v_{1}}{L}-\frac{2 \pi i v_{2}}{L}\right) \\
&\left.-\left(\frac{t}{2 \pi}\right)^{-\frac{2 \pi i v_{2}}{L}} \zeta\left(1-\frac{2 \pi i v_{2}}{L}\right) \zeta\left(1+\frac{2 \pi i v_{2}}{L}\right) A\left(\frac{2 \pi i v_{2}}{L}\right) \frac{\zeta^{\prime}}{\zeta}\left(1+\frac{2 \pi i v_{1}}{L}\right)\right) d t d v_{1} d v_{2} .
\end{aligned}
$$


Keeping only the polar terms and performing the integral over $T$ of the form

$$
\int_{0}^{T}\left(\frac{t}{2 \pi}\right)^{-\frac{2 \pi i v}{L}} d t \sim T e^{-2 \pi i v}
$$

we find (2.80) is asymptotic to

$$
\begin{gathered}
\frac{T}{2 \pi} L \int_{-\infty}^{\infty} \int_{-\infty}^{\infty} f\left(v_{1}, v_{2}\right)\left(-\frac{e^{-2 \pi i v_{1}}}{i(2 \pi)^{3} v_{1}^{2}\left(v_{2}-v_{1}\right)}+\frac{e^{-2 \pi i v_{1}}}{i(2 \pi)^{3} v_{1}^{2} v_{2}}\right. \\
\left.-\frac{e^{-2 \pi i v_{2}}}{i(2 \pi)^{3} v_{2}^{2}\left(v_{1}-v_{2}\right)}+\frac{e^{-2 \pi i v_{2}}}{i(2 \pi)^{3} v_{1} v_{2}^{2}}\right) d v_{1} d v_{2} .
\end{gathered}
$$

A similar calculation can be done for

$$
\frac{1}{L^{2} 2 \pi} \int_{-\frac{T L}{2 \pi}}^{\frac{T L}{2 \pi}} \int_{-\frac{T L}{2 \pi}}^{\frac{T L}{2 \pi}} f\left(v_{1}, v_{2}\right) I\left(0, \frac{2 \pi i v_{1}}{L} ;-\frac{2 \pi i v_{2}}{L}\right) d v_{1} d v_{2}
$$

and it can be seen to be asymptotic to

$$
\begin{aligned}
\frac{T}{2 \pi} L \int_{-\infty}^{\infty} \int_{-\infty}^{\infty} & f\left(v_{1}, v_{2}\right)\left(-\frac{e^{2 \pi i v_{2}}}{i(2 \pi)^{3} v_{1} v_{2}^{2}}+\frac{e^{2 \pi i v_{2}}}{i(2 \pi)^{3} v_{2}^{2}\left(v_{1}-v_{2}\right)}\right. \\
& \left.+\frac{e^{-2 \pi i\left(v_{1}-v_{2}\right)}}{i(2 \pi)^{3} v_{1}\left(v_{1}-v_{2}\right)^{2}}-\frac{e^{-2 \pi i\left(v_{1}-v_{2}\right)}}{i(2 \pi)^{3} v_{2}\left(v_{1}-v_{2}\right)^{2}}\right) d v_{1} d v_{2} .
\end{aligned}
$$

All of the other terms containing a variation of $I\left(\alpha_{1}, \alpha_{2} ; \beta\right)$ can be obtained from (2.83) and (2.85) by a simple swapping of $v_{1}$ and $v_{2}$ or by changing the sign of these variables.

After combining like terms, the exponential terms in the integrands of (2.83) and (2.85) and the other similar integrals sum to

$$
\begin{aligned}
& -\frac{e^{-2 \pi i v_{1}}}{4 i \pi^{3} v_{1}^{2}\left(v_{2}-v_{1}\right)}+\frac{e^{2 \pi i v_{1}}}{4 i \pi^{3} v_{1}^{2}\left(v_{2}-v_{1}\right)}-\frac{e^{2 \pi i v_{1}}}{4 i \pi^{3} v_{1}^{2} v_{2}}+\frac{e^{-2 \pi i v_{1}}}{4 i \pi^{3} v_{1}^{2} v_{2}} \\
& -\frac{e^{-2 \pi i v_{2}}}{4 i \pi^{3} v_{2}^{2}\left(v_{1}-v_{2}\right)}+\frac{e^{2 \pi i v_{2}}}{4 i \pi^{3} v_{2}^{2}\left(v_{1}-v_{2}\right)}+\frac{e^{-2 \pi i v_{2}}}{4 i \pi^{3} v_{1} v_{2}^{2}}-\frac{e^{2 \pi i v_{2}}}{4 i \pi^{3} v_{1} v_{2}^{2}} \\
& \quad-\frac{e^{2 \pi i\left(v_{1}-v_{2}\right)}}{4 i \pi^{3} v_{1}\left(v_{1}-v_{2}\right)^{2}}+\frac{e^{-2 \pi i\left(v_{1}-v_{2}\right)}}{4 i \pi^{3} v_{1}\left(v_{1}-v_{2}\right)^{2}}-\frac{e^{-2 \pi i\left(v_{1}-v_{2}\right)}}{4 i \pi^{3} v_{2}\left(v_{1}-v_{2}\right)^{2}}+\frac{e^{2 \pi i\left(v_{1}-v_{2}\right)}}{4 i \pi^{3} v_{2}\left(v_{1}-v_{2}\right)^{2}} \\
& =\frac{\sin \left(2 \pi v_{1}\right)}{2 \pi^{3} v_{1}^{2}\left(v_{2}-v_{1}\right)}-\frac{\sin \left(2 \pi v_{1}\right)}{2 \pi^{3} v_{1}^{2} v_{2}}+\frac{\sin \left(2 \pi v_{2}\right)}{2 \pi^{3} v_{2}^{2}\left(v_{1}-v_{2}\right)} \\
& \quad-\frac{\sin \left(2 \pi v_{2}\right)}{2 \pi^{3} v_{1} v_{2}^{2}}-\frac{\sin \left(2 \pi\left(v_{1}-v_{2}\right)\right)}{2 \pi^{3} v_{1}\left(v_{1}-v_{2}\right)^{2}}+\frac{\sin \left(2 \pi\left(v_{1}-v_{2}\right)\right)}{2 \pi^{3} v_{2}\left(v_{1}-v_{2}\right)^{2}}
\end{aligned}
$$

Taking a common denominator, we arrive at

$$
\frac{-\sin \left(2 \pi v_{1}\right)+\sin \left(2 \pi v_{2}\right)+\sin \left(2 \pi\left(v_{1}-v_{2}\right)\right)}{2 \pi^{3} v_{1} v_{2}\left(v_{1}-v_{2}\right)},
$$

and use the identity

$$
\sin x \sin y \sin (x-y)=\frac{1}{4}(\sin (2 x-2 y)-\sin (2 x)+\sin (2 y))
$$


to show that all the terms in (2.78) containing $I\left(\alpha_{1}, \alpha_{2} ; \beta\right)$ sum to

$$
\frac{T}{2 \pi} \log \frac{T}{2 \pi} \int_{-\infty}^{\infty} \int_{-\infty}^{\infty} 2 f\left(v_{1}, v_{2}\right) \frac{\sin \left(\pi v_{1}\right)}{\pi v_{1}} \frac{\sin \left(\pi v_{2}\right)}{\pi v_{2}} \frac{\sin \left(\pi\left(v_{1}-v_{2}\right)\right)}{\pi\left(v_{1}-v_{2}\right)} d v_{1} d v_{2}
$$

The $I_{1}(\alpha ; \beta)$ terms are simpler. We start with

$$
\frac{1}{L^{2} 2 \pi} \int_{-\frac{T L}{2 \pi}}^{\frac{T L}{2 \pi}} \int_{-\frac{T L}{2 \pi}}^{\frac{T L}{2 \pi}} f\left(v_{1}, v_{2}\right) I_{1}\left(0 ; \frac{2 \pi i v_{2}}{L}\right) d v_{1} d v_{2} .
$$

Picking out just the relevant terms from (2.3), the leading-order contribution to (2.90) is contained in

$$
\begin{aligned}
\frac{1}{L^{2} 2 \pi} \int_{-\frac{T L}{2 \pi}}^{\frac{T L}{2 \pi}} \int_{-\frac{T L}{2 \pi}}^{\frac{T L}{2 \pi}} f\left(v_{1}, v_{2}\right) \int_{0}^{T} \log \frac{t}{2 \pi}\left(\left(\frac{\zeta^{\prime}}{\zeta}\right)^{\prime}\left(1+\frac{2 \pi i v_{2}}{L}\right)\right. \\
\left.\quad+\left(\frac{t}{2 \pi}\right)^{-\frac{2 \pi i v_{2}}{L}} \zeta\left(1+\frac{2 \pi i v_{2}}{L}\right) \zeta\left(1-\frac{2 \pi i v_{2}}{L}\right) A\left(\frac{2 \pi i v_{2}}{L}\right)\right) d t d v_{1} d v_{2} .
\end{aligned}
$$

Expanding the zeta functions around their pole and keeping just the leading-order term, we then perform the integration over $T$ and arrive at the asymptotic result for (2.90) for large $T$

$$
-\frac{T}{2 \pi} \log \frac{T}{2 \pi} \int_{-\infty}^{\infty} \int_{-\infty}^{\infty} f\left(v_{1}, v_{2}\right)\left(\frac{1}{\left(2 \pi v_{2}\right)^{2}}-\frac{e^{-2 \pi i v_{2}}}{\left(2 \pi v_{2}\right)^{2}}\right) d v_{1} d v_{2}
$$

We combine this with

$$
\begin{aligned}
& \frac{1}{L^{2} 2 \pi} \int_{-\frac{T L}{2 \pi}}^{\frac{T L}{2 \pi}} \int_{-\frac{T L}{2 \pi}}^{\frac{T L}{2 \pi}} f\left(v_{1}, v_{2}\right) I_{1}\left(-\frac{2 \pi i v_{2}}{L} ; 0\right) d v_{1} d v_{2} \\
& \sim-\frac{T}{2 \pi} \log \frac{T}{2 \pi} \int_{-\infty}^{\infty} \int_{-\infty}^{\infty} f\left(v_{1}, v_{2}\right)\left(\frac{1}{\left(2 \pi v_{2}\right)^{2}}-\frac{e^{2 \pi i v_{2}}}{\left(2 \pi v_{2}\right)^{2}}\right) d v_{1} d v_{2}
\end{aligned}
$$

and the sum gives

$$
\begin{gathered}
-\frac{T}{2 \pi} \log \frac{T}{2 \pi} \int_{-\infty}^{\infty} \int_{-\infty}^{\infty} f\left(v_{1}, v_{2}\right)\left(\frac{1}{2\left(\pi v_{2}\right)^{2}}-\frac{\cos \left(2 \pi v_{2}\right)}{2\left(\pi v_{2}\right)^{2}}\right) d v_{1} d v_{2} \\
=-\frac{T}{2 \pi} \log \frac{T}{2 \pi} \int_{-\infty}^{\infty} \int_{-\infty}^{\infty} f\left(v_{1}, v_{2}\right) \frac{\sin ^{2}\left(\pi v_{2}\right)}{\left(\pi v_{2}\right)^{2}} d v_{1} d v_{2} .
\end{gathered}
$$

Since $I_{1}(\alpha ; \beta)$ only depends on the sum of its two arguments, we see immediately that in the $T \rightarrow \infty$ limit the $I_{1}$ terms are responsible for $-\frac{\sin ^{2}\left(\pi\left(v_{1}-v_{2}\right)\right)}{\pi^{2}\left(v_{1}-v_{2}\right)^{2}}-\frac{\sin ^{2}\left(\pi v_{1}\right)}{\pi^{2} v_{1}^{2}}-\frac{\sin ^{2}\left(\pi v_{2}\right)}{\pi^{2} v_{2}^{2}}$ in (2.77).

\section{RANDom Matrix Theory}

We now use a very similar method to that in Section 2 to derive the triple correlation of eigenvalues of random unitary matrices. Of course, there are more elegant methods to do this in random matrix theory (see Section 3.1) but the point of Section 3.2 is that it helps to illuminate the preceding calculation of the triple correlation of the Riemann zeros. 
If $X$ is an $N \times N$ matrix with complex entries $X=\left(x_{j k}\right)$, we let $X^{*}$ be its conjugate transpose, i.e. $X^{*}=\left(y_{j k}\right)$ where $y_{j k}=\overline{x_{k j}}$. $X$ is said to be unitary if $X X^{*}=I$. We let $U(N)$ denote the group of all $N \times N$ unitary matrices. This is a compact Lie group and has a Haar measure which allows us to do analysis.

All of the eigenvalues of $X \in U(N)$ have absolute value 1 ; we write them as

$$
e^{i \theta_{1}}, e^{i \theta_{2}}, \ldots, e^{i \theta_{N}} .
$$

For any sequence of $N$ points on the unit circle there are matrices in $U(N)$ with these points as eigenvalues. The collection of all matrices with the same set of eigenvalues constitutes a conjugacy class in $U(N)$. Thus, the set of conjugacy classes can identified with the collection of sequences of $N$ points on the unit circle.

Weyl's formula asserts that for a function $f: U(N) \rightarrow \mathbf{C}$ which is constant on conjugacy classes,

$$
\int_{U(N)} f(X) d \text { Haar }=\int_{[0,2 \pi]^{N}} f\left(\theta_{1}, \ldots, \theta_{N}\right) d X_{N},
$$

where

$$
d X_{N}=\prod_{1 \leq j<k \leq N}\left|e^{i \theta_{k}}-e^{i \theta_{j}}\right|^{2} \frac{d \theta_{1} \ldots d \theta_{N}}{N !(2 \pi)^{N}} .
$$

Since $N$ will be fixed in this paper, we will usually write $d X$ in place of $d X_{N}$.

The characteristic polynomial of a matrix $X$ is denoted $\Lambda_{X}(s)$ and is defined by

$$
\Lambda_{X}(s)=\operatorname{det}\left(I-s X^{*}\right)=\prod_{n=1}^{N}\left(1-s e^{-i \theta_{n}}\right) .
$$

The roots of $\Lambda_{X}(s)$ are the eigenvalues of $X$. The characteristic polynomial satisfies the functional equation

$$
\begin{aligned}
\Lambda_{X}(s) & =(-s)^{N} \prod_{n=1}^{N} e^{-i \theta_{n}} \prod_{n=1}^{N}\left(1-e^{i \theta_{n}} / s\right) \\
& =(-1)^{N} \operatorname{det} X^{*} s^{N} \Lambda_{X^{*}}(1 / s) .
\end{aligned}
$$

Note that

$$
s \frac{\Lambda_{X}^{\prime}}{\Lambda_{X}}(s)+\frac{1}{s} \frac{\Lambda_{X^{*}}^{\prime}}{\Lambda_{X^{*}}}\left(\frac{1}{s}\right)=N .
$$

These characteristic polynomials have value distributions similar to that of the Riemann zetafunction and form the basis of random matrix models which predict behavior for the Riemann zeta-function based on what can be proven about $\Lambda$. Some care has to be taken in making these comparisons because we are used to thinking about the zeta-function in a half-plane whereas the characteristic polynomials are naturally studied on a circle. The translation is that the $1 / 2$-line corresponds to the unit circle; the half-plane to the right of the $1 / 2$-line corresponds to the inside of the unit circle. Note that $\Lambda_{X}(0)=1$ is the analogue of $\lim _{\sigma \rightarrow \infty} \zeta(\sigma+i t)=1$. 
We let

$$
z(x)=\frac{1}{1-e^{-x}} .
$$

In our formulas for averages of characteristic polynomials the function $z(x)$ plays the role for random matrix theory that $\zeta(1+x)$ plays in the theory of moments of the Riemann zeta-function.

3.1. Triple correlation by Gaudin's Lemma. Let $f(x, y, z)$ be a smooth function which is periodic with period $2 \pi$ in each variable. We want a formula for

$$
T_{3}(f):=\int_{U(N)} \sum_{j_{1}, j_{2}, j_{3}}^{*} f\left(\theta_{j_{1}}, \theta_{j_{2}}, \theta_{j_{3}}\right) d X,
$$

where the sum is for distinct indices $j_{1}, j_{2}, j_{3}$. It is a standard result in random matrix theory that by Gaudin's Lemma (see, for example, [21] or [9]), we have

$$
T_{3}(f)=\frac{1}{(2 \pi)^{3}} \int_{[0,2 \pi]^{3}} f\left(\theta_{1}, \theta_{2}, \theta_{3}\right) \operatorname{det}_{3 \times 3} S_{N}\left(\theta_{k}-\theta_{j}\right) d \theta_{3} d \theta_{2} d \theta_{1}
$$

where

$$
S_{N}(\theta)=\frac{\sin \frac{N \theta}{2}}{\sin \frac{\theta}{2}}
$$

3.2. Triple correlation via the ratios theorem. We now produce an alternate method of calculation of the triple correlation for eigenvalues of unitary matrices. This method mirrors that produced earlier in the paper for the Riemann zeros, but many steps are cleaner and more obvious in the random matrix case, not to mention the fact that they are all rigorous. Therefore the calculation in this section serves to clarify and support the previous calculation of the triple correlation of the Riemann zeros.

Let

$$
g(z):=\Lambda_{X}\left(e^{z}\right)=\prod_{j=1}^{N}\left(1-e^{z} e^{-i \theta_{j}}\right) .
$$

Then, since $g(z)$ has zeros at $z=i \theta_{j}+2 \pi i m, m \in \mathbb{Z}$, by Cauchy's theorem we have for an arbitrary holomorphic, $2 \pi i$ periodic function $f$,

$$
\sum_{j=1}^{N} f\left(\theta_{j}\right)=\frac{1}{2 \pi i} \int_{\mathcal{C}} \frac{g^{\prime}}{g}(z) f(z / i) d z=\frac{1}{2 \pi i} \int_{\mathcal{C}} e^{z} \frac{\Lambda_{X}^{\prime}}{\Lambda_{X}}\left(e^{z}\right) f(z / i) d z,
$$

where $\mathcal{C}$ is a positively oriented contour which encloses a subinterval of the imaginary axis of length $2 \pi$. We choose a specific path $\mathcal{C}$ to be the positively oriented rectangle that has vertices $\delta-\pi i, \delta+\pi i,-\delta+\pi i,-\delta-\pi i$ where $\delta$ is a small positive number. Note that, by periodicity, the integrals on the horizontal segments cancel each other. Applying this three times, and replacing 
each variable by its negative, we have (using the fact that $\mathcal{C}$ is unchanged when $z \rightarrow-z$ ),

$$
\begin{aligned}
& M_{3}(f):= \int_{U(N)} \sum_{j_{1}=1}^{N} \sum_{j_{2}=1}^{N} \sum_{j_{3}=1}^{N} f\left(\theta_{j_{1}}, \theta_{j_{2}}, \theta_{j_{3}}\right) d X \\
&= \frac{-1}{(2 \pi i)^{3}} \int_{\mathcal{C}} \int_{\mathcal{C}} \int_{\mathcal{C}} e^{-z_{1}-z_{2}-z_{3}} \int_{U(N)} \frac{\Lambda_{X}^{\prime}}{\Lambda_{X}}\left(e^{-z_{1}}\right) \frac{\Lambda_{X}^{\prime}}{\Lambda_{X}}\left(e^{-z_{2}}\right) \frac{\Lambda_{X}^{\prime}}{\Lambda_{X}}\left(e^{-z_{3}}\right) d X \\
& \quad \times f\left(i z_{1}, i z_{2}, i z_{3}\right) d z_{3} d z_{2} d z_{1}
\end{aligned}
$$

for a three variable holomorphic periodic function $f$. Notice that $M_{3}(f)$ is like $T_{3}(f)$ except that it is a sum over all triples $\left(j_{1}, j_{2}, j_{3}\right)$ of indices between 1 and $N$ instead of over distinct indices.

Let $\mathcal{C}_{-}$denote the path along the left side of $\mathcal{C}$ from $-\delta+\pi i$ down to $-\delta-\pi i$ and let $\mathcal{C}_{+}$denote the path along the right side of $\mathcal{C}$ from $\delta-\pi i$ up to $\delta+\pi i$. Ignoring the integrals over the horizontal paths (because their contribution is 0 ) we take each variable $z_{j}$ to be on one or the other of the two vertical paths $\mathcal{C}_{-}$or $\mathcal{C}_{+}$. In this way our expression can be written as a sum of eight terms, each term being a triple integral with each integral on a vertical line segment either $\mathcal{C}_{-}$or $\mathcal{C}_{+}$. These eight integrals are analogous to $J_{1}, \ldots, J_{8}$ in Section 2.3. As we did for the Riemann zeta function, for each variable $z_{j}$ which is on $\mathcal{C}_{-}$we use the functional equation (3.6) to replace $e^{-z_{j}} \frac{\Lambda_{X}^{\prime}}{\Lambda_{X}}\left(e^{-z_{j}}\right)$ by $N-e^{z_{j}} \frac{\Lambda_{X^{*}}^{\prime}}{\Lambda_{X^{*}}}\left(e^{z_{j}}\right)$. In this way we find (using $X^{-1}=X^{*}$ ) that

$$
\begin{aligned}
M_{3}(f)= & \frac{-1}{(2 \pi i)^{3}} \sum_{\substack{\epsilon_{j} \in\{-1,+1\} \\
j=1,2,3}} \int_{\mathcal{C}_{\epsilon_{1}}} \int_{\mathcal{C}_{\epsilon_{2}}} \int_{\mathcal{C}_{\epsilon_{3}}} \int_{U(N)} \prod_{j=1}^{3}\left(\frac{1-\epsilon_{j}}{2} N+\epsilon_{j} e^{-\epsilon_{j} z_{j}} \frac{\Lambda_{X^{\epsilon_{j}}}^{\prime}}{\Lambda_{X^{\epsilon_{j}}}}\left(e^{-\epsilon_{j} z_{j}}\right)\right) d X \\
& \times f\left(i z_{1}, i z_{2}, i z_{3}\right) d z_{3} d z_{2} d z_{1} .
\end{aligned}
$$

We next compute the averages over $X \in U(N)$ which appear in the above equation.

We need two instances of the ratios theorem (see [12] or [13 for statements and proofs of this theorem). Recall the definition of $z(x)$ from (3.7).

(A) Let $\Re \gamma, \Re \delta>0$.

$$
\int_{U(N)} \frac{\Lambda_{X}\left(e^{-\alpha}\right) \Lambda_{X^{*}}\left(e^{-\beta}\right)}{\Lambda_{X}\left(e^{-\gamma}\right) \Lambda_{X^{*}}\left(e^{-\delta}\right)} d X=\frac{z(\alpha+\beta) z(\gamma+\delta)}{z(\alpha+\delta) z(\beta+\gamma)}+e^{-N(\alpha+\beta)} \frac{z(-\beta-\alpha) z(\gamma+\delta)}{z(-\beta+\delta) z(-\alpha+\gamma)} .
$$

(B) Let $\Re \gamma_{1}, \Re \gamma_{2}, \Re \delta>0$. Then

$$
\begin{gathered}
\int_{U(N)} \frac{\Lambda_{X}\left(e^{-\alpha_{1}}\right) \Lambda_{X}\left(e^{-\alpha_{2}}\right) \Lambda_{X^{*}}\left(e^{-\beta}\right)}{\Lambda_{X}\left(e^{-\gamma_{1}}\right) \Lambda_{X}\left(e^{-\gamma_{2}}\right) \Lambda_{X^{*}}\left(e^{-\delta}\right)} d X=\frac{z\left(\alpha_{1}+\beta\right) z\left(\alpha_{2}+\beta\right) z\left(\gamma_{1}+\delta\right) z\left(\gamma_{2}+\delta\right)}{z\left(\alpha_{1}+\delta\right) z\left(\alpha_{2}+\delta\right) z\left(\beta+\gamma_{1}\right) z\left(\beta+\gamma_{2}\right)} \\
+e^{-N\left(\alpha_{1}+\beta\right)} \frac{z\left(-\beta-\alpha_{1}\right) z\left(\alpha_{2}-\alpha_{1}\right) z\left(\gamma_{1}+\delta\right) z\left(\gamma_{2}+\delta\right)}{z(-\beta+\delta) z\left(\alpha_{2}+\delta\right) z\left(-\alpha_{1}+\gamma_{1}\right) z\left(-\alpha_{1}+\gamma_{2}\right)} \\
+e^{-N\left(\alpha_{2}+\beta\right)} \frac{z\left(-\beta-\alpha_{2}\right) z\left(\alpha_{1}-\alpha_{2}\right) z\left(\gamma_{1}+\delta\right) z\left(\gamma_{2}+\delta\right)}{z(-\beta+\delta) z\left(\alpha_{1}+\delta\right) z\left(-\alpha_{2}+\gamma_{1}\right) z\left(-\alpha_{2}+\gamma_{2}\right)} .
\end{gathered}
$$

For application to triple correlation, we need averages of logarithmic derivatives of the characteristic polynomials. Differentiating the above formulas leads to the following two identities, which 
are the random matrix version of (2.3) and (2.2) respectively, remembering that $z(x)$ plays the role of $\zeta(1+x)$ and $N$ the role of $\log \frac{t}{2 \pi}$. The products and sums of primes in (2.3) and (2.2) do not have any counterpart in random matrix theory.

Proposition 3.1. If $\Re \alpha, \Re \beta>0$, then

$$
\begin{aligned}
J(\alpha ; \beta): & =e^{-\alpha-\beta} \int_{U(N)} \frac{\Lambda_{X}^{\prime}}{\Lambda_{X}}\left(e^{-\alpha}\right) \frac{\Lambda_{X^{*}}^{\prime}}{\Lambda_{X^{*}}}\left(e^{-\beta}\right) d X \\
& =\left(\frac{z^{\prime}}{z}\right)^{\prime}(\alpha+\beta)+e^{-N(\alpha+\beta)} z(\alpha+\beta) z(-\alpha-\beta) .
\end{aligned}
$$

Proposition 3.2. Let $\Re \alpha_{1}, \Re \alpha_{2}, \Re \beta>0$. Then

$$
\begin{aligned}
J\left(\alpha_{1}, \alpha_{2} ; \beta\right):= & -e^{-\alpha_{1}-\alpha_{2}-\beta} \int_{U(N)} \frac{\Lambda_{X}^{\prime}}{\Lambda_{X}}\left(e^{-\alpha_{1}}\right) \frac{\Lambda_{X}^{\prime}}{\Lambda_{X}}\left(e^{-\alpha_{2}}\right) \frac{\Lambda_{X^{*}}^{\prime}}{\Lambda_{X^{*}}}\left(e^{-\beta}\right) d X \\
= & e^{-N\left(\alpha_{1}+\beta\right)} z\left(\alpha_{1}+\beta\right) z\left(-\alpha_{1}-\beta\right)\left(\frac{z^{\prime}}{z}\left(\alpha_{2}-\alpha_{1}\right)-\frac{z^{\prime}}{z}\left(\alpha_{2}+\beta\right)\right) \\
& \quad+e^{-N\left(\alpha_{2}+\beta\right)} z\left(\alpha_{2}+\beta\right) z\left(-\alpha_{2}-\beta\right)\left(\frac{z^{\prime}}{z}\left(\alpha_{1}-\alpha_{2}\right)-\frac{z^{\prime}}{z}\left(\alpha_{1}+\beta\right)\right) .
\end{aligned}
$$

Note that the right-hand-side has a simple pole when $\alpha_{1}=-\beta$ and when $\alpha_{2}=-\beta$ but is analytic when $\alpha_{1}=\alpha_{2}$. We also remark that non-constant integrals with no $X$, or no $X^{*}$, are 0 ; for example, if $\Re \alpha, \Re \beta>0$, then

$$
\int_{U(N)} \frac{\Lambda_{X}^{\prime}}{\Lambda_{X}}\left(e^{-\alpha}\right) d X=\int_{U(N)} \frac{\Lambda_{X^{*}}^{\prime}}{\Lambda_{X^{*}}}\left(e^{-\alpha}\right) d X=\int_{U(N)} \frac{\Lambda_{X}^{\prime}}{\Lambda_{X}}\left(e^{-\alpha}\right) \frac{\Lambda_{X}^{\prime}}{\Lambda_{X}}\left(e^{-\beta}\right) d X=0
$$

Thus, letting $d G$ be a shorthand for $f\left(i z_{1}, i z_{2}, i z_{3}\right) d z_{3} d z_{2} d z_{1}$, we have, upon expanding (3.14),

$$
\begin{aligned}
-(2 \pi i)^{3} M_{3}(f)=N^{3} \int_{\mathcal{C}_{-}} \int_{\mathcal{C}_{-}} \int_{\mathcal{C}_{-}} d G+\int_{\mathcal{C}_{+}} \int_{\mathcal{C}_{+}} \int_{\mathcal{C}_{-}} J\left(z_{1}, z_{2} ;-z_{3}\right) d G \\
\quad+\int_{\mathcal{C}_{-}} \int_{\mathcal{C}_{+}} \int_{\mathcal{C}_{+}} J\left(z_{2}, z_{3} ;-z_{1}\right) d G+\int_{\mathcal{C}_{+}} \int_{\mathcal{C}_{-}} \int_{\mathcal{C}_{+}} J\left(z_{1}, z_{3} ;-z_{2}\right) d G \\
-\int_{\mathcal{C}_{-}} \int_{\mathcal{C}_{-}} \int_{\mathcal{C}_{+}}\left(J\left(-z_{1},-z_{2} ; z_{3}\right)+N\left(J\left(-z_{1} ; z_{3}\right)+J\left(-z_{2} ; z_{3}\right)\right) d G\right. \\
-\int_{\mathcal{C}_{-}} \int_{\mathcal{C}_{+}} \int_{\mathcal{C}_{-}}\left(J\left(-z_{1},-z_{3} ; z_{2}\right)+N\left(J\left(-z_{1} ; z_{2}\right)+J\left(-z_{3} ; z_{2}\right)\right) d G\right. \\
-\int_{\mathcal{C}_{+}} \int_{\mathcal{C}_{-}} \int_{\mathcal{C}_{-}}\left(J\left(-z_{2},-z_{3} ; z_{1}\right)+N\left(J\left(-z_{2} ; z_{1}\right)+J\left(-z_{3} ; z_{1}\right)\right) d G .\right.
\end{aligned}
$$

This is very similar to (2.53), except that we have retained three variables instead of working with the differences $z_{2}-z_{1}$ and $z_{3}-z_{1}$. 
Now, by the inclusion-exclusion principle,

$$
\begin{aligned}
T_{3}(f)=M_{3}(f) & -\int_{U(N)} \sum_{j_{1}, j_{2}}\left(f\left(\theta_{j_{1}}, \theta_{j_{1}}, \theta_{j_{2}}\right)+f\left(\theta_{j_{1}}, \theta_{j_{2}}, \theta_{j_{1}}\right)+f\left(\theta_{j_{1}}, \theta_{j_{2}}, \theta_{j_{2}}\right)\right) d X \\
& +2 \int_{U(N)} \sum_{j} f\left(\theta_{j}, \theta_{j}, \theta_{j}\right) d X
\end{aligned}
$$

The pair-correlation sums are evaluated much as above. For example,

$$
\begin{gathered}
\int_{U(N)} \sum_{j_{1}, j_{3}} f\left(\theta_{j_{1}}, \theta_{j_{3}}, \theta_{j_{3}}\right) d X=\frac{1}{(2 \pi i)^{2}}\left(-\int_{\mathcal{C}_{-}} \int_{\mathcal{C}_{+}} J\left(-z_{1} ; z_{3}\right) f\left(i z_{1}, i z_{3}, i z_{3}\right) d z_{3} d z_{1}\right. \\
-\int_{\mathcal{C}_{+}} \int_{\mathcal{C}_{-}} J\left(z_{1} ;-z_{3}\right) f\left(i z_{1}, i z_{3}, i z_{3}\right) d z_{3} d z_{1} \\
\left.+N^{2} \int_{\mathcal{C}_{-}} \int_{\mathcal{C}_{-}} f\left(i z_{1}, i z_{3}, i z_{3}\right) d z_{2} d z_{1}\right) .
\end{gathered}
$$

The one-correlation sum is

$$
\int_{U(N)} \sum_{j \leq N} f\left(\theta_{j}, \theta_{j}, \theta_{j}\right) d X=\frac{-N}{2 \pi i} \int_{\mathcal{C}_{-}} f(i z, i z, i z) d z .
$$

Now we move all of the paths of integration over to the imaginary axis. When we do this we encounter some poles and have to use principal value integrals. For example,

$$
\begin{aligned}
\int_{\mathcal{C}_{+}} \int_{\mathcal{C}_{+}} \int_{\mathcal{C}_{-}} J\left(z_{1}, z_{2} ;-z_{3}\right) d G \\
=\int_{-\pi i}^{\pi i} \int_{\mathcal{C}_{+}} \int_{\mathcal{C}_{-}} J\left(z_{1}, z_{2} ;-z_{3}\right) d G \\
=\int_{-\pi i}^{\pi i} \lim _{\epsilon \rightarrow 0^{+}} \int_{\substack{[-\pi i, \pi i] \\
\left|z_{2}-z_{1}\right|>\epsilon}} \int_{\mathcal{C}_{-}} J\left(z_{1}, z_{2} ;-z_{3}\right) d G \\
=-\int_{-\pi i}^{\pi i} \lim _{\epsilon \rightarrow 0^{+}} \int_{\substack{[-\pi i, \pi i] \\
\left|z_{2}-z_{1}\right|>\epsilon}} \lim _{\delta \rightarrow 0^{+}} \int_{\substack{\left|z_{3}-z_{2}\right|>\delta \\
\left|z_{3}-z_{1}\right|>\delta}} J\left(z_{1}, z_{2} ;-z_{3}\right) d G \\
\quad+\pi i \int_{-\pi i}^{\pi i} \lim _{\epsilon \rightarrow 0^{+}} \int_{\substack{[-\pi i, \pi i] \\
\left|z_{2}-z_{1}\right|>\epsilon}} \operatorname{Res}_{z_{3}=z_{1}}\left(J\left(z_{1}, z_{2} ;-z_{3}\right) f\left(i z_{1}, i z_{2}, i z_{3}\right)\right) d z_{2} d z_{1} \\
\quad+\pi i \int_{-\pi i}^{\pi i} \lim _{\epsilon \rightarrow 0^{+}} \int_{\substack{[-\pi i, \pi i] \\
\left|z_{2}-z_{1}\right|>\epsilon}} \operatorname{Res}_{z_{3}=z_{2}}\left(J\left(z_{1}, z_{2} ;-z_{3}\right) f\left(i z_{1}, i z_{2}, i z_{3}\right)\right) d z_{2} d z_{1} .
\end{aligned}
$$

The first equality follows because there are no singularities when we move $z_{1}$ onto the imaginary axis. In the second, there are again no singularities when we move $z_{2}$ onto the imaginary axis, but anticipating what comes next we choose to write this integral with a limit as $\epsilon \rightarrow 0^{+}$. Finally, the third equality takes into account the poles at $z_{1}$ and $z_{2}$ when we move $z_{3}$ onto the imaginary axis. To determine the signs of the three terms in the final equality, remember the the contour $\mathcal{C}_{-}$ is oriented downwards and just to the left of the imaginary axis. Thus when it is moved onto the 
vertical axis it wraps the singularities in the positive (anti-clockwise) direction. Also the residues are multiplied by $\pi i$ instead of $2 \pi i$ because we moved the path onto a path that goes right through the singularities, i.e. we have not crossed the poles but rather moved on top of them, and so we have only one-half the usual contribution of a residual term and the integral in $z_{3}$ that remains in the first term of the final equality is a principal value integral.

Now it is easily calculated that

$$
\underset{z_{3}=z_{1}}{\operatorname{Res}} J\left(z_{1}, z_{2} ;-z_{3}\right)=-J\left(z_{2} ;-z_{1}\right) \quad \text { and } \quad \operatorname{Res}_{z_{3}=z_{2}} J\left(z_{1}, z_{2} ;-z_{3}\right)=-J\left(z_{1} ;-z_{2}\right) \text {. }
$$

Thus, we have

$$
\begin{aligned}
\int_{\mathcal{C}_{+}} \int_{\mathcal{C}_{+}} \int_{\mathcal{C}_{-}} J\left(z_{1}, z_{2} ;-z_{3}\right) d G \\
=-\int_{-\pi i}^{\pi i} \lim _{\epsilon \rightarrow 0^{+}} \int_{\substack{[-\pi i, \pi i] \\
\left|z_{2}-z_{1}\right|>\epsilon}} \lim _{\delta \rightarrow 0^{+}} \int_{\substack{[-\pi i, \pi i] \\
\left|z_{3}-z_{2}\right|>\delta \\
\left|z_{3}-z_{1}\right|>\delta}} J\left(z_{1}, z_{2} ;-z_{3}\right) d G \\
\quad-\pi i \int_{-\pi i}^{\pi i} \lim _{\epsilon \rightarrow 0^{+}} \int_{\substack{[-\pi i, \pi i] \\
\left|z_{2}-z_{1}\right|>\epsilon}} J\left(z_{2} ;-z_{1}\right) f\left(i z_{1}, i z_{2}, i z_{1}\right) d z_{2} d z_{1} \\
\quad-\pi i \int_{-\pi i}^{\pi i} \lim _{\epsilon \rightarrow 0^{+}} \int_{\substack{[-\pi i, \pi i] \\
\left|z_{2}-z_{1}\right|>\epsilon}} J\left(z_{1} ;-z_{2}\right) f\left(i z_{1}, i z_{2}, i z_{2}\right) d z_{2} d z_{1} .
\end{aligned}
$$

A slightly different calculation gives

$$
\begin{aligned}
& \int_{\mathcal{C}_{-}} \int_{\mathcal{C}_{+}} \int_{\mathcal{C}_{+}} J\left(z_{3}, z_{2} ;-z_{1}\right) d G \\
& =-\int_{-\pi i}^{\pi i} \int_{\mathcal{C}_{+}} \int_{\mathcal{C}_{+}} J\left(z_{3}, z_{2} ;-z_{1}\right) d G \\
& =-\int_{-\pi i}^{\pi i} \lim _{\epsilon \rightarrow 0^{+}} \int_{\substack{[-\pi i, \pi i] \\
\left|z_{2}-z_{1}\right|>\epsilon}} \int_{\mathcal{C}_{+}} J\left(z_{3}, z_{2} ;-z_{1}\right) d G \\
& +\pi i \int_{-\pi i}^{\pi i} \operatorname{Res}_{z_{2}=z_{1}}\left(\int_{\mathcal{C}_{+}} J\left(z_{3}, z_{2} ;-z_{1}\right) f\left(i z_{1}, i z_{2}, i z_{3}\right) d z_{3}\right) d z_{1} \\
& =-\int_{-\pi i}^{\pi i} \lim _{\epsilon \rightarrow 0^{+}} \int_{\substack{[-\pi i, \pi i] \\
\left|z_{2}-z_{1}\right|>\epsilon}} \int_{\mathcal{C}_{+}} J\left(z_{3}, z_{2} ;-z_{1}\right) d G \\
& +\pi i \int_{-\pi i}^{\pi i} \int_{\mathcal{C}_{+}} J\left(z_{3} ;-z_{1}\right) f\left(i z_{1}, i z_{1}, i z_{3}\right) d z_{3} d z_{1}
\end{aligned}
$$


after moving $z_{1}$ and $z_{2}$ onto the imaginary axis. Now we move $z_{3}$ onto the imaginary axis and obtain a residual term from each of the two integrals above. Thus,

$$
\begin{aligned}
\int_{\mathcal{C}_{-}} \int_{\mathcal{C}_{+}} & \int_{\mathcal{C}_{+}} J\left(z_{3}, z_{2} ;-z_{1}\right) d G \\
=- & \int_{-\pi i}^{\pi i} \lim _{\epsilon \rightarrow 0^{+}} \int_{\substack{[-\pi i, \pi i] \\
\left|z_{2}-z_{1}\right|>\epsilon}} \lim _{\delta \rightarrow 0^{+}} \int_{\substack{[-\pi i, \pi i] \\
\left|z_{3}-z_{2}\right|>\delta \\
\left|z_{3}-z_{1}\right|>\delta}} J\left(z_{3}, z_{2} ;-z_{1}\right) d G \\
& -\pi i \int_{-\pi i}^{\pi i} \lim _{\epsilon \rightarrow 0^{+}} \int_{\substack{[-\pi i, \pi i] \\
\left|z_{2}-z_{1}\right|>\epsilon}} \operatorname{Res}_{z_{3}=z_{1}}\left(J\left(z_{3}, z_{2} ;-z_{1}\right) f\left(i z_{1}, i z_{2}, i z_{3}\right)\right) d z_{2} d z_{1} \\
& -\pi i \int_{-\pi i}^{\pi i} \lim _{\epsilon \rightarrow 0^{+}} \int_{\substack{[-\pi i, \pi i] \\
\left|z_{3}-z_{1}\right|>\epsilon}} J\left(z_{3} ;-z_{1}\right) f\left(i z_{1}, i z_{1}, i z_{3}\right) d z_{3} d z_{1} \\
& -(\pi i)^{2} \int_{-\pi i}^{\pi i} \operatorname{Res}_{z_{3}=z_{1}}\left(J\left(z_{3} ;-z_{1}\right) f\left(i z_{1}, i z_{1}, i z_{3}\right)\right) d z_{1} .
\end{aligned}
$$

Now

$$
\underset{z_{3}=z_{1}}{\operatorname{Res}} J\left(z_{3}, z_{2} ;-z_{1}\right)=-J\left(z_{2} ;-z_{1}\right) \quad \text { and } \quad \underset{z_{3}=z_{1}}{\operatorname{Res}} J\left(z_{3} ;-z_{1}\right)=-N \text {. }
$$

Thus, we end up with

$$
\begin{aligned}
\int_{\mathcal{C}_{-}} \int_{\mathcal{C}_{+}} & \int_{\mathcal{C}_{+}} J\left(z_{3}, z_{2} ;-z_{1}\right) d G \\
= & -\int_{-\pi i}^{\pi i} \lim _{\epsilon \rightarrow 0^{+}} \int_{\substack{[-\pi i, \pi i] \\
\left|z_{2}-z_{1}\right|>\epsilon}} \lim _{\delta \rightarrow 0^{+}} \int_{\substack{|[-\pi i, \pi i]\\
| z_{3}-z_{2}|>\delta\\
| z_{3}-z_{1} \mid>\delta}} J\left(z_{3}, z_{2} ;-z_{1}\right) d G \\
& -\pi i \int_{-\pi i}^{\pi i} \lim _{\epsilon \rightarrow 0^{+}} \int_{\substack{[-\pi i, \pi i] \\
\left|z_{2}-z_{1}\right|>\epsilon}} J\left(z_{2} ;-z_{1}\right) f\left(i z_{1}, i z_{2}, i z_{1}\right) d z_{2} d z_{1} \\
& -\pi i \int_{-\pi i}^{\pi i} \lim _{\substack{\mid-\pi 0^{+} \\
\int_{\mid-\pi i, \pi i]}}} J\left(z_{3} ;-z_{1}\right) f\left(i z_{1}, i z_{1}, i z_{3}\right) d z_{3} d z_{1} \\
& -(\pi i)^{2} N \int_{-\pi i}^{\pi i} f(i z, i z, i z) d z .
\end{aligned}
$$

By a change of variables we have

$$
\int_{\mathcal{C}_{+}} \int_{\mathcal{C}_{-}} \int_{\mathcal{C}_{-}} J\left(-z_{3},-z_{2} ; z_{1}\right) d G=-\int_{\mathcal{C}_{-}} \int_{\mathcal{C}_{+}} \int_{\mathcal{C}_{+}} J\left(z_{3}, z_{2} ;-z_{1}\right) d G .
$$

We can also make use of the symmetry $J\left(\alpha_{1}, \alpha_{2} ; \beta\right)=J\left(\alpha_{2}, \alpha_{1} ; \beta\right)$. In this way we can use (3.25) and (3.28) to replace all of the integrals involving a three-variable $J$ in terms of principal value integrals. 
In a similar way we rewrite, for example, the two variable $J$ - integral as

$$
\begin{gathered}
\int_{\mathcal{C}_{+}} \int_{\mathcal{C}_{-}} J\left(z_{1} ;-z_{3}\right) f\left(i z_{1}, i z_{3}, i z_{3}\right) d z_{3} d z_{1} \\
=-\int_{-\pi i \epsilon \rightarrow 0^{+}}^{\pi i} \int_{\substack{\mid-\pi i, \pi i] \\
\left|z_{3}-z_{1}\right|>\epsilon}} J\left(z_{1} ;-z_{3}\right) f\left(i z_{1}, i z_{3}, i z_{3}\right) d z_{3} d z_{1} \\
\quad-\pi i N \int_{-\pi i}^{\pi i} f(i z, i z, i z) d z .
\end{gathered}
$$

Using (3.14) - (3.30), after the substitutions $i z_{j}=\theta_{j}, j=1,2,3$, and some simplification, we have

$$
\begin{aligned}
& T_{3}(f)= \frac{1}{(2 \pi)^{3}} \int_{-\pi}^{\pi} \lim _{\epsilon \rightarrow 0^{+}} \int_{\substack{[-\pi, \pi] \\
\left|\theta_{2}-\theta_{1}\right|>\epsilon}} \lim _{\delta \rightarrow 0^{+}} \int_{\substack{[-\pi, \pi] \\
\left|\theta_{3}-\theta_{1}\right|>\delta \\
\theta_{3}-\theta_{2} \mid>\delta}}\left(J\left(i \theta_{1}, i \theta_{2} ;-i \theta_{3}\right)+J\left(i \theta_{1}, i \theta_{3} ;-i \theta_{2}\right)\right. \\
&+J\left(i \theta_{2}, i \theta_{3} ;-i \theta_{1}\right)+J\left(-i \theta_{1},-i \theta_{2} ; i \theta_{3}\right)+J\left(-i \theta_{1},-i \theta_{3} ; i \theta_{2}\right)+J\left(-i \theta_{2},-i \theta_{3} ; i \theta_{1}\right) \\
&+N\left(J\left(-i \theta_{1} ; i \theta_{3}\right)+J\left(-i \theta_{2} ; i \theta_{3}\right)+J\left(-i \theta_{1} ; i \theta_{2}\right)+J\left(-i \theta_{3} ; i \theta_{2}\right)\right. \\
&\left.\left.\quad+J\left(-i \theta_{2} ; i \theta_{1}\right)+J\left(-i \theta_{3} ; i \theta_{1}\right)\right)+N^{3}\right) f\left(\theta_{1}, \theta_{2}, \theta_{3}\right) d \theta_{1} d \theta_{2} d \theta_{3},
\end{aligned}
$$

all of the pair-correlation and one-correlation terms having cancelled. The principal value integrals with the limits in $\epsilon$ and $\delta$ are no longer needed because it can be checked, using Mathematica for example, that the integrand here is

$$
=\operatorname{det}_{3 \times 3} S_{N}\left(\theta_{k}-\theta_{j}\right) f\left(\theta_{1}, \theta_{2}, \theta_{3}\right)
$$

which is entire. Now our formula agrees with (3.9).

This concludes our alternate proof of triple correlation for unitary matrices.

\section{REFERENCES}

[1] M.V. Berry, Semiclassical formula for the number variance of the Riemann zeros, Nonlinearity, 1:399-407, 1988.

[2] M.V. Berry and J.P. Keating, The Riemann zeros and eigenvalue asymptotics, SIAM Rev., 41(2):236-266, 1999.

[3] E.B. Bogomolny, Spectral statistics and periodic orbits, In New Directions in Quantum Chaos; editors, G. Casati, I. Guarneri, and U. Smilansky, pages 333-369. IOS Press, Amsterdam, 2000.

[4] E.B. Bogomolny and J.P. Keating, private communication.

[5] E.B. Bogomolny and J.P. Keating, Random matrix theory and the Riemann zeros I: three- and four-point correlations, Nonlinearity, 8:1115-1131, 1995.

[6] E.B. Bogomolny and J.P. Keating, Gutzwiller's trace formula and spectral statistics: beyond the diagonal approximation, Phys. Rev. Lett., 77(8):1472-1475, 1996.

[7] E.B. Bogomolny and J.P. Keating, Random matrix theory and the Riemann zeros II: $n$-point correlations, Nonlinearity, 9:911-935, 1996.

[8] J.B. Conrey, L-functions and random matrices, In Mathematics Unlimited 2001 and Beyond; editors, B. Enquist and W. Schmid, pages 331-352. Springer-Verlag, Berlin, 2001, arXiv math.nt/0005300.

[9] J.B. Conrey, Notes on eigenvalue distributions for the classical compact groups, In Recent perspectives on random matrix theory and number theory, LMS Lecture Note Series 322, pages 111-45. Cambridge University Press, Cambridge, 2005.

[10] J.B. Conrey, D.W. Farmer, J.P. Keating, M.O. Rubinstein and N.C. Snaith, Integral moments of $L$-functions, Proc. Lond. Math. Soc., 91, No. 1, pages 33-104, 2005, arXiv math.nt/0206018.

[11] J.B. Conrey, D.W. Farmer, and M.R. Zirnbauer, Autocorrelation of ratios of characteristic polynomials and of $L$-functions, in preparation. 
[12] J.B. Conrey, D.W. Farmer, and M.R. Zirnbauer, Howe pairs, supersymmetry, and ratios of random characteristic polynomials for the unitary groups $U(N)$, preprint, arXiv math-ph/0511024.

[13] J.B. Conrey, P.J. Forrester, and N.C. Snaith, Averages of ratios of characteristic polynomials for the compact classical groups, Int. Math. Res. Notices, 7:397-431, 2005.

[14] J.B. Conrey and N.C. Snaith, Applications of the L-functions ratios conjectures, Proc. Lon. Math. Soc. 94(3):594646, 2007, arXiv math.NT/0509480

[15] G.H. Hardy and J.E. Littlewood, Contributions to the theory of the Riemann zeta-function and the theory of the distribution of primes, Acta Mathematica, 41:119-196, 1918.

[16] D.A. Hejhal, On the triple correlation of zeros of the zeta function, Inter. Math. Res. Notices, 7:293-302, 1994.

[17] J.P. Keating, The Riemann zeta function and quantum chaology, In Quantum Chaos; editors, G. Casati, I Guarneri, and U. Smilansky, pages 145-85. North-Holland, Amsterdam, 1993.

[18] J.P. Keating, Periodic orbits, spectral statistics, and the Riemann zeros, In Supersymmetry and trace formulae: chaos and disorder; editors, I.V. Lerner, J.P. Keating, and D.E. Khmelnitskii, pages 1-15. Plenum, New York, 1999.

[19] J.P. Keating and N.C. Snaith, Random matrix theory and $\zeta(1 / 2+i t)$, Commun. Math. Phys., 214:57-89, 2000.

[20] J.P. Keating and N.C. Snaith, Random matrices and L-functions, J. Phys. A, 36(12):2859-81, 2003.

[21] M.L. Mehta, Random Matrices, Academic Press, London, second edition, 1991.

[22] H.L. Montgomery, The pair correlation of the zeta function, Proc. Symp. Pure Math, 24:181-93, 1973.

[23] Z. Rudnick and P. Sarnak, Zeros of principal L-functions and random matrix theory, Duke Mathematical Journal, 81(2):269-322, 1996.

American Institute of Mathematics, 360 Portage Ave, Palo Alto, CA 94306

School of Mathematics, University of Bristol, Bristol, BS8 1TW, United Kingdom

E-mail address: conrey@aimath.org

School of Mathematics, University of Bristol, Bristol, BS8 1TW, United Kingdom

E-mail address: N.C.Snaith@bris.ac.uk 